\title{
OVERLAPPING SELF-AFFINE SETS OF KAKEYA TYPE
}

\author{
ANTTI KÄENMÄKI AND PABLO SHMERKIN
}

\begin{abstract}
We compute the Minkowski dimension for a family of self-affine sets on $\mathbb{R}^{2}$. Our result holds for every (rather than generic) set in the class. Moreover, we exhibit explicit open subsets of this class where we allow overlapping, and do not impose any conditions on the norms of the linear maps. The family under consideration was inspired by the theory of Kakeya sets.
\end{abstract}

\section{INTRODUCTION}

An iterated function system (IFS) on $\mathbb{R}^{d}$ is a finite collection of strictly contractive self-maps $f_{1}, \ldots, f_{\kappa}$. A classical result, formalized by Hutchinson [1] (although the crucial idea goes back to Moran [18]), states that for every IFS there is a unique nonempty compact set $E \subset \mathbb{R}^{d}$ for which

$$
E=\bigcup_{i=1}^{\kappa} f_{i}(E) \text {. }
$$

When the mappings are similitudes (or conformal) and the pieces $f_{i}(E)$ do not overlap much, the Hausdorff dimension of $E$ is easily determined by the contraction ratios of the mappings $f_{i}$, see for example [11, [16, and [14]. In the present article, we assume that the mappings $f_{i}$ are affine; in this case the set $E$ is called a self-affine set. In addition, we do not require any non-overlapping condition. Dropping either the conformality or separation hypothesis makes the problem of estimating dimension dramatically more complicated. The main feature of our work is that we are able to drop both, while obtaining results which are valid everywhere, not just generically.

The so-called singular value function plays a prominent rôle in the study of the dimension of self-affine sets. Following [3, Proposition 4.1], the singular value function leads to a notion of the singular value dimension, which serves as an upper bound for the upper Minkowski dimension, see [1] and [3]. Falconer [3] (see also [22]) proved that assuming the norms of the linear parts to be less than $\frac{1}{2}$, this

Date: November 15, 2018.

2000 Mathematics Subject Classification. Primary 28A80; Secondary 37C45.

Key words and phrases. Kakeya set, self-affine set, Minkowski dimension.

AK acknowledges the support of the Academy of Finland (project \#114821). Research of PS was partially supported by NSF grant \#DMS-0355187 and the Academy of Finland. Part of this research was carried out while PS was visiting the Instituto de Matemática Pura e Aplicada (IMPA), Brazil. 
upper bound is sharp, and also equals the Hausdorff dimension, for $\mathcal{L}^{d \kappa}$-almost every choice of translation vectors. Here $\mathcal{L}^{d \kappa}$ denotes the Lebesgue measure on $\mathbb{R}^{d \kappa}$. Falconer and Miao [7] have recently shown that the size of the set of exceptional translation vectors is small also in the sense of Hausdorff dimension. The self-affine carpets of McMullen [17] show that one cannot replace "almost all" by "all", even if the pieces do not overlap. Furthermore, it follows from examples in [2] that the $\frac{1}{2}$ bound on the norms is essential. These counterexamples are of a very special kind, and it is therefore of interest to find families of self-affine sets for which one can loose these assumptions.

A result into this direction was obtained by Hueter and Lalley in [10], where it is proven that for an explicit open class of self-affine sets, the Hausdorff dimension is indeed given by the singular value dimension, as long as the pieces $f_{i}(E)$ are disjoint. In their result the norms may be greater than $\frac{1}{2}$, but it follows from their hypotheses that the singular value dimension is less than 1 . In a different direction, it was recently proven in [12] that for a randomized version of self-affine sets the natural analogue of Falconer's formula holds almost surely regardless of the norms. See also [9], [13], [8], [20], and [6] for other recent results on the dimensional properties of self-affine sets.

For a fixed $\kappa$ and $d$, the class of all IFSs consisting of $\kappa$ affine maps on $\mathbb{R}^{d}$ inherits a natural topology from $\mathcal{A}_{d}^{\kappa}$, where $\mathcal{A}_{d}=G L_{d}(\mathbb{R}) \times \mathbb{R}^{d}$ is identified with the vector space of all invertible affine mappings on $\mathbb{R}^{d}$. We will say that a family of affine IFS's is robust if it is open in this topology, and that a property is stable if the set of IFS's where it holds is robust.

We define a class of self-affine sets in which we allow overlapping and the norms of all the maps can be arbitrarily close to 1 ; see $\oint 3$ for the details. We show that in this class the Minkowski dimension coincides with the singular value dimension (Theorem 3.3), and it can be defined dynamically as the zero of a certain pressure function. Even though the family is not itself robust, in $\$ 6$ we will exhibit robust subsets which preserve all the interesting properties. This is the first instance where the equality of Minkowski dimension and singular value dimension is established for a robust family, without requiring any separation assumptions. Moreover, we prove that the Minkowski dimension is a continuous function of the generating maps within this family.

The inspiration for our work arose from the theory of Kakeya sets. Recall that a subset of $\mathbb{R}^{d}$ is called a Kakeya set (sometimes also a Besicovitch set) if it contains a unit segment in every direction. The long-standing Kakeya conjecture asserts, in one of its many forms, that the Hausdorff dimension of a Kakeya set in $\mathbb{R}^{d}$ is precisely $d$. This is wide open for $d \geq 3$; however, for $d=2$ it is known to be true, and indeed the proof is not difficult, see for example [25]. This result implies that the overlap between segments pointing in different directions is small, in the sense that the dimension of the union of all segments is the same as if there was no overlap at all. We strove to construct a family of self-affine sets 
in which the cylinder sets are aligned in different directions, so that the possible overlaps between them would not affect the dimension calculations. Although the technical details may obscure it somewhat, it may be useful to keep this basic idea in mind while going through the definitions and proofs.

The paper is structured as follows. In 92 , we introduce some standard notation and present some preliminary facts on self-affine sets. The family of self-affine sets of Kakeya type is defined in \$3, where Theorem 3.3, the main result of the paper, is stated. The proof of Theorem 3.3 is contained in \$4. In \$5, we study projections of self-affine sets, as part of our preparation to obtain explicit examples of self-affine sets of Kakeya-type. These examples are introduced in $₫ 6$, where we finish our discussion with some remarks and open questions.

\section{SELF-AFFINE SETS}

Throughout the article, we use the following notation: Let $0<\bar{\alpha}<1$ and $I=\{1, \ldots, \kappa\}$ with $\kappa \geq 2$. Put $I^{*}=\bigcup_{n=1}^{\infty} I^{n}$ and $I^{\infty}=I^{\mathbb{N}}$. For each $i \in I^{*}$, there is $n \in \mathbb{N}$ such that $\mathrm{i}=\left(i_{1}, \ldots, i_{n}\right) \in I^{n}$. We call this $n$ as the length of $i$ and we denote $|i|=n$. The length of elements in $I^{\infty}$ is infinity. Moreover, if $i \in I^{*}$ and $j \in I^{*} \cup I^{\infty}$ then with the notation $i j$ we mean the element obtained by juxtaposing the terms of $i$ and $j$. For $i \in I^{*}$, we define $[i]=\left\{i j: j \in I^{\infty}\right\}$ and we call the set $[i]$ a cylinder set of level $|i|$. If $j \in I^{*} \cup I^{\infty}$ and $1 \leq n<|j|$, we define $\left.j\right|_{n}$ to be the unique element $i \in I^{n}$ for which $j \in[i]$. We also denote $i^{-}=\left.i\right|_{|i|-1}$. With the notation $i \perp j$, we mean that the elements $i, j \in I^{*}$ are incomparable, that is, $[\mathrm{i}] \cap[\mathrm{j}]=\emptyset$. We call a set $A \subset I^{*}$ incomparable if all of its elements are mutually incomparable. Finally, with the notation $i \wedge j$, we mean the common beginning of $i \in I^{*}$ and $j \in I^{*}$, that is, $i \wedge j=\left.i\right|_{n}=\left.j\right|_{n}$, where $n=\min \left\{k-1:\left.i\right|_{k} \neq\left. j\right|_{k}\right\}$.

Defining

$$
|i-j|= \begin{cases}\bar{\alpha}^{|i \wedge j|}, & i \neq j \\ 0, & i=j\end{cases}
$$

whenever $i, j \in I^{\infty}$, the couple $\left(I^{\infty},|\cdot|\right)$ is a compact metric space. We call $\left(I^{\infty},|\cdot|\right)$ a symbol space and an element $i=\left(i_{1}, i_{2}, \ldots\right) \in I^{\infty}$ a symbol. If there is no danger of misunderstanding, we will also call an element $i \in I^{*}$ a symbol. Define the left shift $\sigma: I^{\infty} \rightarrow I^{\infty}$ by setting

$$
\sigma\left(i_{1}, i_{2}, \ldots\right)=\left(i_{2}, i_{3}, \ldots\right)
$$

The notation $\sigma\left(i_{1}, \ldots, i_{n}\right)$ means the symbol $\left(i_{2}, \ldots, i_{n}\right) \in I^{n-1}$. Observe that to be precise in our definitions, we need to work with "empty symbols", that is, symbols with zero length, which will be denoted by $\varnothing$.

The singular values $1>\|A\|=\alpha_{1}(A) \geq \cdots \geq \alpha_{d}(A)>0$ of a contractive invertible matrix $A \in \mathbb{R}^{d \times d}$ are the square roots of the eigenvalues of the positive 
definite matrix $A^{*} A$, where $A^{*}$ is the transpose of $A$. The normalized eigenvectors of $A^{*} A$ are denoted by $\theta_{1}(A), \ldots, \theta_{d}(A)$. These eigenvectors together with singular values give geometric information about the matrix $A$. For example, let $v$ be the unit vector with direction equal to the major axis of the ellipse $A(B)$, where $B$ is any ball. By definition, the direction of $v$ is the image under $A$ of a vector which maximizes $|A x|$ over all $x$ in the unit ball. But $\theta_{1}(A)$ is precisely such a vector since $|A x|^{2}=A^{*} A x \cdot x$. Thus, explicitly, $v=A\left(\theta_{1}(A)\right) / \alpha_{1}(A)$. For more detailed information, the reader is referred to [24, §V.1.3].

For a contractive invertible matrix $A \in \mathbb{R}^{d \times d}$, we define the singular value function to be

$$
\varphi^{t}(A)=\alpha_{1}(A) \cdots \alpha_{l}(A) \alpha_{l+1}(A)^{t-l},
$$

where $0 \leq t<d$ and $l$ is the integer part of $t$. For $t \geq d$, we put $\varphi^{t}(A)=$ $\left(\alpha_{1}(A) \cdots \alpha_{d}(A)\right)^{t / d}=|\operatorname{det}(A)|^{t / d}$.

For each $i \in I$, fix a contractive invertible matrix $A_{i} \in \mathbb{R}^{d \times d}$ such that $\left\|A_{i}\right\| \leq$ $\bar{\alpha}<1$. Clearly the products $A_{\mathrm{i}}=A_{i_{1}} \cdots A_{i_{n}}$ are also contractive and invertible as $i \in I^{n}$ and $n \in \mathbb{N}$. Denoting $\underline{\alpha}=\min _{i \in I} \alpha_{d}\left(A_{i}\right)>0$, for each $t, \delta \geq 0$ we have

$$
\varphi^{t}\left(A_{\dot{\mathbf{i}}}\right) \underline{\alpha}^{\delta|\mathbf{i}|} \leq \varphi^{t+\delta}\left(A_{\mathrm{i}}\right) \leq \varphi^{t}\left(A_{\mathbf{i}}\right) \bar{\alpha}^{\delta|\mathbf{i}|}
$$

whenever $i \in I^{*}$. According to [24, Corollary V.1.1] and [3, Lemma 2.1], the following holds for all $t \geq 0$ :

$$
\varphi^{t}\left(A_{\mathrm{ij}}\right) \leq \varphi^{t}\left(A_{\mathrm{i}}\right) \varphi^{t}\left(A_{\mathrm{j}}\right)
$$

whenever $i, j \in I^{*}$.

Given $t \geq 0$, we define the topological pressure to be

$$
P(t)=\lim _{n \rightarrow \infty} \frac{1}{n} \log \sum_{\mathrm{i} \in I^{n}} \varphi^{t}\left(A_{\mathrm{i}}\right) .
$$

The limit above exists by the standard theory of subadditive sequences since for each $t \geq 0$, using (2.3),

$$
\sum_{\mathbf{i} \in I^{n+m}} \varphi^{t}\left(A_{\mathbf{i}}\right) \leq \sum_{\mathbf{i} \in I^{n+m}} \varphi^{t}\left(A_{\left.\mathbf{i}\right|_{n}}\right) \varphi^{t}\left(A_{\sigma^{n}(\mathbf{i})}\right)=\sum_{\mathbf{i} \in I^{n}} \varphi^{t}\left(A_{\mathbf{i}}\right) \sum_{\mathbf{j} \in I^{m}} \varphi^{t}\left(A_{\mathbf{j}}\right)
$$

whenever $n, m \in \mathbb{N}$. Moreover, as a function, $P:[0, \infty) \rightarrow \mathbb{R}$ is continuous and strictly decreasing with $\lim _{t \rightarrow \infty} P(t)=-\infty$ : For $t, \delta \geq 0$ and $n \in \mathbb{N}$, we have, using (2.2),

$$
\delta \log \underline{\alpha}+\frac{1}{n} \log \sum_{\mathbf{i} \in I^{n}} \varphi^{t}\left(A_{\mathbf{i}}\right) \leq \frac{1}{n} \log \sum_{\mathbf{i} \in I^{n}} \varphi^{t+\delta}\left(A_{\mathbf{i}}\right) \leq \delta \log \bar{\alpha}+\frac{1}{n} \log \sum_{\mathbf{i} \in I^{n}} \varphi^{t}\left(A_{\mathbf{i}}\right) .
$$

Letting $n \rightarrow \infty$, we get $0<-\delta \log \bar{\alpha} \leq P(t)-P(t+\delta) \leq-\delta \log \underline{\alpha}$. Since $P(0)=\log \kappa$, we have actually shown that there exists a unique $t>0$ for which $P(t)=0$. The singular value dimension is defined to be the zero of the topological pressure. See also [3, Proposition 4.1]. 
Theorem 2.1. Suppose that for each $i \in I$ there is an invertible matrix $A_{i} \in$ $\mathbb{R}^{d \times d}$ with $\left\|A_{i}\right\| \leq \bar{\alpha}$. If for given $t \geq 0$ there exists a constant $D \geq 1$ such that

$$
D^{-1} \varphi^{t}\left(A_{\mathrm{i}}\right) \varphi^{t}\left(A_{\mathrm{j}}\right) \leq \varphi^{t}\left(A_{\mathrm{ij}}\right)
$$

whenever $\mathrm{i}, \mathrm{j} \in I^{*}$ then there exists a Borel probability measure $\mu$ on $I^{\infty}$, a constant $c \geq 1$, and $1>\lambda_{1}(\mu) \geq \cdots \geq \lambda_{d}(\mu)>0$ such that

$$
c^{-1} e^{-|\mathbf{i}| P(t)} \varphi^{t}\left(A_{\mathbf{i}}\right) \leq \mu([\mathbf{i}]) \leq c e^{-|\mathbf{i}| P(t)} \varphi^{t}\left(A_{\mathbf{i}}\right)
$$

whenever $i \in I^{*}$ and

$$
\lim _{n \rightarrow \infty} \alpha_{k}\left(A_{\left.i\right|_{n}}\right)^{1 / n}=\lambda_{k}(\mu)
$$

for $\mu$-almost all $i \in I^{\infty}$ and for every $k \in\{1, \ldots, d\}$.

Proof. Using the assumptions, (2.2), and (2.3), the existence of a Borel probability measure $\mu$ satisfying (2.5) follows from [14, Theorem 2.2] by a minor modification. More precisely, in [14] it was assumed that the parameter $t$ is an exponent, but an examination of the proof reveals that this fact is not required. Using [14, Theorem 2.2], (2.3), and Kingman's subadditive ergodic theorem [23], the limit

$$
E^{t}(\mu)=\lim _{n \rightarrow \infty} \frac{1}{n} \log \varphi^{t}\left(A_{\left.i\right|_{n}}\right)
$$

exists for $\mu$-almost every $i \in I^{*}$ and for every $t \geq 0$. Setting now $\lambda_{k}(\mu)=$ $\exp \left(E^{k}(\mu)-E^{k-1}(\mu)\right)$ for $k \in\{1, \ldots, d\}$, we have finished the proof.

It may appear that the assumption of Theorem 2.1 is very strong. However, it is implied by some simple geometrical conditions; see Remark 4.2. Observe also that even if the measure satisfying (2.5) did not exist, the latter claim of Theorem 2.1 remains true for the natural measure found in [13, Theorem 4.1].

If for each $i \in I$ an invertible matrix $A_{i} \in \mathbb{R}^{d \times d}$ with $\left\|A_{i}\right\| \leq \bar{\alpha}$ and a translation vector $a_{i}$ are fixed then we define a projection mapping $\pi: I^{\infty} \rightarrow \mathbb{R}^{d}$ by setting

$$
\pi(\mathrm{i})=\sum_{n=1}^{\infty} A_{\left.\mathrm{i}\right|_{n-1}} a_{i_{n}}
$$

as $\mathbf{i}=\left(i_{1}, i_{2}, \ldots\right)$. Using the triangle inequality, we have

$$
\begin{aligned}
|\pi(\mathrm{i})-\pi(\mathrm{j})| & \leq \sum_{n=|\mathbf{i} \wedge \mathrm{j}|+1}^{\infty}\left|A_{\left.\mathrm{i}\right|_{n-1}} a_{i_{n}}-A_{\left.\mathrm{j}\right|_{n-1}} a_{j_{n}}\right| \\
& \leq \sum_{n=|\mathbf{i} \wedge \mathrm{j}|+1}^{\infty} 2 \bar{\alpha}^{n-1} \max _{i \in I}\left|a_{i}\right|=\frac{2 \max _{i \in I}\left|a_{i}\right|}{1-\bar{\alpha}}|\mathbf{i}-\mathrm{j}|
\end{aligned}
$$

for every $i, j \in I^{\infty}$. The mapping $\pi$ is therefore continuous. 
We define $E=\pi\left(I^{\infty}\right)$ and call this set a self-affine set. Observe that the compact set $E$ is invariant under the affine mappings $A_{i}+a_{i}$, that is,

$$
E=\bigcup_{i=1}^{\kappa}\left(A_{i}+a_{i}\right)(E)
$$

This is an immediate consequence of the fact that

$$
\pi(i \mathrm{i})=\left(A_{i}+a_{i}\right) \sum_{n=1}^{\infty} A_{\left.\mathrm{i}\right|_{n-1}} a_{i_{n}}=\left(A_{i}+a_{i}\right) \pi(\mathrm{i})
$$

whenever $i \in I^{\infty}$ and $i \in I$. In fact, by [11, §3.1], there are no other nonempty compact sets satisfying (2.6) besides $E$. If there is no danger of misunderstanding, the image of a cylinder set

$$
\pi([\mathbf{i}])=\left(A_{i_{1}}+a_{i_{1}}\right) \cdots\left(A_{i_{n}}+a_{i_{n}}\right)(E)=A_{\mathbf{i}}(E)+A_{\left.\mathbf{i}\right|_{n-1}} a_{i_{n}}+\cdots+a_{i_{1}},
$$

as $\mathrm{i}=\left(i_{1}, \ldots, i_{n}\right) \in I^{n}$, will also be called a cylinder set, and we will denote $E_{\mathrm{i}}=\pi([\mathrm{i}])$. When we want to emphasize the dependence of $E$ on the affine mappings, we will say that $E$ is the invariant set of the affine IFS $\left\{A_{i}+a_{i}\right\}_{i \in I}$.

\section{Self-Affine sets of Kakeya type}

In this section, we introduce self-affine sets of Kakeya type. Working in $\mathbb{R}^{2}$, we state that the Minkowski dimension of such a set is the zero of the topological pressure, see (2.4). Given a set $A \subset \mathbb{R}^{d}$, the upper and lower Minkowski dimensions are denoted by $\overline{\operatorname{dim}}_{\mathrm{M}}(A)$ and $\underline{\operatorname{dim}}_{\mathrm{M}}(A)$, respectively. For the definition, see [15, §5.3]. If $\overline{\operatorname{dim}}_{\mathrm{M}}(A)=\underline{\operatorname{dim}}_{\mathrm{M}}(A)$, then the common value, the Minkowski dimension, is denoted by $\operatorname{dim}_{\mathrm{M}}(A)$. For $\theta \in S^{d-1}=\left\{x \in \mathbb{R}^{d}:|x|=1\right\}$ and $0 \leq \beta \leq \pi$, we set

$$
X(\theta, \beta)=\left\{x \in \mathbb{R}^{d}: \cos (\beta / 2)<|\theta \cdot x| /|x|, x \neq 0\right\} .
$$

The closure of a given set $A$ is denoted by $\bar{A}$ and with the notation $\mathcal{L}^{d}$, we mean the Lebesgue measure on $\mathbb{R}^{d}$.

Definition 3.1. Suppose that for each $i \in I$ there are a contractive invertible matrix $A_{i} \in \mathbb{R}^{2 \times 2}$ with $\left\|A_{i}\right\| \leq \bar{\alpha}<1$ and a translation vector $a_{i} \in \mathbb{R}^{2}$. The collection of affine mappings $\left\{A_{i}+a_{i}\right\}_{i \in I}$ is called an affine iterated function system of Kakeya type, and the invariant set $E \subset \mathbb{R}^{2}$ of this affine IFS a selfaffine set of Kakeya type, provided that the following two conditions hold:

(K1) There exist $\theta \in S^{1}$ and $0<\beta<\pi / 2$ such that

$$
\begin{array}{ll}
A_{i}(\overline{X(\theta, \beta)}) \subset X(\theta, \beta), & (\underline{\mathrm{K} 1 \mathrm{a})} \\
A_{i}^{*}(\overline{X(\theta, \beta)}) \subset X(\theta, \beta) & \text { (1ㅏ) }
\end{array}
$$

whenever $i \in I$ and

$$
A_{i}(\overline{X(\theta, \beta)}) \cap A_{j}(\overline{X(\theta, \beta)})=\{0\}
$$


for $i \neq j$.

(K2) There exists a constant $\varrho>0$ such that

$$
\mathcal{L}^{1}\left(\left\{\theta_{1}\left(A_{\mathrm{i}}\right) \cdot x: x \in E\right\}\right) \geq \varrho
$$

for all $i \in I^{*}$.

Let us make some remarks on these conditions. Our goal is to make the selfaffine set look, at a given finite scale, roughly like a rescaled Kakeya set (except that instead of having segments in every direction, there are segments only in a Cantor set of directions). The rôle of the conditions $(\underline{\mathrm{K} 1 \mathrm{a}})$ and $(\underline{\mathrm{K} 1 \mathrm{C}})$ is to ensure that cylinder sets are aligned in different directions. Notice the analogy between these conditions and the Hypothesis 3 ("separation") in [10]. The hypothesis

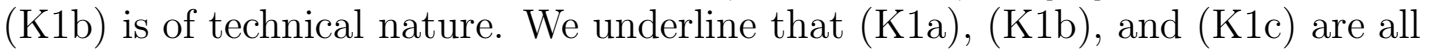
stable properties.

The projection condition $(\underline{\mathrm{K} 2})$ is needed so that cylinder sets do not have too many "holes" and one can approximate them by neighborhoods of segments. It is the only one of the assumptions which involves the translation vectors $\left\{a_{i}\right\}_{i \in I}$ in addition to the linear maps $\left\{A_{i}\right\}_{i \in I}$. In particular, (K2) implies that the Hausdorff dimension of $E$ is at least one. Hence if $t$ is such that $P(t)=0$, then $t \geq 1$ by [3, Proposition 5.1]. An analogous, but stronger, projection condition was introduced by Falconer in [4]. We remark that in that article, unlike in our case, the open set condition is also required. The projection condition is obviously satisfied if the invariant set is connected. Unfortunately, determining when a self-affine set is connected in a stable way is a very difficult problem, even when the linear parts commute, see for example [21]. In \$5, we introduce easily checkable, stable conditions which imply the projection condition.

We do not need analogues of either Hypothesis 2 ("distortion") or Hypothesis 5 ("strong separation") used in [10]. In that article, Hypothesis 2 plays a crucial rôle in guaranteeing that the invariant set has dimension less than 1. By our observation that $t \geq 1$, it cannot possibly hold in our setting. In a sense, our examples are more purely self-affine, since both singular values are involved in the dimension calculations, while in [10] the dimension depends only on the largest one. We stress that our results are only for the Minkowski dimension; estimating the Hausdorff dimension in our setting appears to be a very difficult problem.

Before stating our main result, we formulate and prove a Kakeya-type estimate which is a crucial ingredient of the proof. Even though it is a minor variant of [25. Proposition 1.5], complete details are provided for the convenience of the reader.

Proposition 3.2. Let $R_{1}, \ldots, R_{M} \subset \mathbb{R}^{2}$ be rectangles of size $\alpha_{1} \times \alpha_{2}$, with $\alpha_{1}>\alpha_{2}$. Suppose that the angle between the long sides of any two rectangles is at least $\alpha_{2} / \alpha_{1}$. If $F \subset \mathbb{R}^{2}$ and $\tau>0$ are such that $\mathcal{L}^{2}\left(F \cap R_{i}\right) \geq \tau \alpha_{1} \alpha_{2}$ for 
every $i \in\{1, \ldots, M\}$, then

$$
\mathcal{L}^{2}(F) \geq \frac{M \tau^{2} \alpha_{1} \alpha_{2}}{2 \sqrt{2} \pi \log \left(2 \pi \alpha_{1} / \alpha_{2}\right)}
$$

Proof. Given two rectangles $R_{i}$ and $R_{j}$, let us denote the (smaller) angle between their long sides by $\Varangle\left(R_{i}, R_{j}\right)$. Since $\alpha_{2} / \alpha_{1} \leq \Varangle\left(R_{i}, R_{j}\right) \leq \pi / 2$, a simple geometric inspection yields $\alpha_{2} / \alpha_{1}+\Varangle\left(R_{i}, R_{j}\right) \leq 2 \Varangle\left(R_{i}, R_{j}\right) \leq \sqrt{2} \pi \sin \left(\Varangle\left(R_{i}, R_{j}\right) / 2\right)=$ $\sqrt{2} \pi \alpha_{2} / \operatorname{diam}\left(R_{i} \cap R_{j}\right)$ and hence

$$
\mathcal{L}^{2}\left(R_{i} \cap R_{j}\right) \leq \alpha_{2} \operatorname{diam}\left(R_{i} \cap R_{j}\right) \leq \frac{\sqrt{2} \pi \alpha_{2}^{2}}{\alpha_{2} / \alpha_{1}+\Varangle\left(R_{i}, R_{j}\right)}
$$

whenever $i \neq j$. Thus we have

$$
\sum_{j=1}^{M} \mathcal{L}^{2}\left(R_{i} \cap R_{j}\right) \leq \sum_{j=0}^{\left\lceil\frac{\pi \alpha_{1}}{2 \alpha_{2}}\right\rceil} \frac{\sqrt{2} \pi \alpha_{2}^{2}}{\alpha_{2} / \alpha_{1}+j \alpha_{2} / \alpha_{1}} \leq 2 \sqrt{2} \pi \alpha_{1} \alpha_{2} \log \left(2 \pi \alpha_{1} / \alpha_{2}\right)
$$

whenever $i \in\{1, \ldots, M\}$. Here with the notation $\lceil x\rceil$, we mean the smallest integer greater than $x$. Since, by using Hölder's inequality,

$$
\begin{aligned}
\left(M \tau \alpha_{1} \alpha_{2}\right)^{2} & \leq\left(\sum_{i=1}^{M} \mathcal{L}^{2}\left(F \cap R_{i}\right)\right)^{2}=\left(\int_{\mathbb{R}^{2}} \chi_{F} \sum_{i=1}^{M} \chi_{R_{i}} d \mathcal{L}^{2}\right)^{2} \\
& \leq\left(\int_{\mathbb{R}^{2}} \chi_{F}^{2} d \mathcal{L}^{2}\right)\left(\int_{\mathbb{R}^{2}}\left(\sum_{i=1}^{M} \chi_{R_{i}}\right)^{2} d \mathcal{L}^{2}\right) \\
& =\mathcal{L}^{2}(F) \sum_{i=1}^{M} \sum_{j=1}^{M} \mathcal{L}^{2}\left(R_{i} \cap R_{j}\right),
\end{aligned}
$$

the claim follows by applying (3.2). Here $\chi_{A}$ denotes the characteristic function of a given set $A$.

We can now state the main result of this article.

Theorem 3.3. Suppose $E \subset \mathbb{R}^{2}$ is a self-affine set of Kakeya type and $P(t)=0$. Then

$$
\operatorname{dim}_{\mathrm{M}}(E)=t \geq 1 \text {. }
$$

In particular, $\operatorname{dim}_{\mathrm{M}}$ is a continuous function when restricted to the class of affine IFS's of Kakeya-type.

Let us sketch the main idea of the proof; full details are postponed until $\S 4$, In order to compute the Minkowski dimension, we want to estimate the area of the set $E(\delta)$ for small $\delta>0$, where $E(\delta)$ is the $\delta$-neighborhood of $E$. In order to do this we take a small $r$ and decompose $E$ as a union of cylinders $\left\{E_{\mathrm{i}}\right\}$ with $\varphi^{t}\left(A_{\mathrm{i}}\right) \approx r$ (where $t$ is the singularity dimension). The condition (K2) implies 
that the projection of $E_{\mathrm{i}}$ onto the major axis of the ellipse $A_{i} B+a_{i}$ (where $B$ is some large ball) has positive Lebesgue measure with a uniform lower bound. Hence it follows that for large $K$ the $K \alpha_{2}\left(A_{\mathrm{i}}\right)$-neighborhood of $E_{\mathrm{i}}$ intersects a rectangle $R_{\mathrm{i}}$, with small side comparable to $\alpha_{2}\left(A_{\mathrm{i}}\right)$ and long side comparable to $\alpha_{1}\left(A_{\mathrm{i}}\right)$, in a set of area comparable to $\alpha_{1}\left(A_{\mathrm{i}}\right) \alpha_{2}\left(A_{\mathrm{i}}\right)$.

At this point we would like to apply the Kakeya-type estimate of Proposition 3.2. However, for this we need all the rectangles to have the same sizes, while $\alpha_{1}\left(A_{\dot{i}}\right)$ and $\alpha_{2}\left(A_{\dot{i}}\right)$ may take many different values. We deal with this with the help of Theorem 2.1; with respect to the measure $\mu$ given by that theorem, the values of $\alpha_{1}\left(A_{\mathrm{i}}\right)$ and $\alpha_{2}\left(A_{\mathrm{i}}\right)$ are roughly constant for "most" sequences $i$. More precisely, we will obtain that $\alpha_{k}\left(A_{\mathbf{i}}\right) \approx\left(\varphi^{t}\left(A_{\mathbf{i}}\right)\right)^{\gamma_{k}}$ for many sequences $\mathrm{i}$, where $\gamma_{1}+(t-1) \gamma_{2}=1$. Also, due to the Gibbs property of $\mu$ expressed in (2.5), the number of cylinders $[\mathrm{i}]$ with $\varphi^{t}\left(A_{\mathrm{i}}\right) \approx r$ is comparable to $r^{-1}$.

By ( $(\underline{\mathrm{K}} \mathrm{l} \mathrm{c})$, the angle between the long sides of two of the rectangles $R_{i}$ and $R_{j}$ in the construction are sufficiently separated. Hence we can apply Proposition 3.2 and conclude that the union of all such rectangles has Lebesgue measure which is, up to a logarithmic factor, the same as if the union was disjoint. Therefore, letting $\delta \approx r^{\gamma_{2}}$ we conclude

$$
\mathcal{L}^{2}(E(\delta)) \gtrsim r^{\gamma_{1}+\gamma_{2}-1+\varepsilon} \approx \delta^{2-t+\varepsilon}
$$

where $\varepsilon>0$ is arbitrarily small, which gives the desired lower estimate (the upper estimate is well known). The latter claim of the theorem is now an immediate consequence of the next lemma.

Lemma 3.4. Suppose that for each $i \in I$ there is a contractive invertible matrix

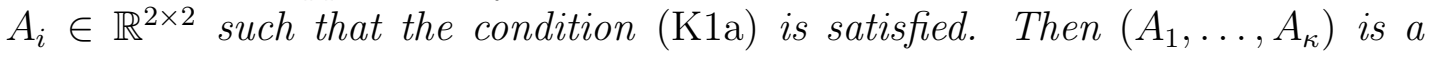
continuity point for the singular value dimension.

Proof. After an appropriate rotation we can assume, without loss of generality, that $\theta=\frac{1}{\sqrt{2}}(1,1)$ in the condition ( $\underline{1} 1$ a $)$. This implies that for each $i \in I$, the coefficients of $A_{i}$ are either all strictly positive or all strictly negative, and this property is preserved under small perturbations. Since multiplying by the scalar -1 does not affect the singular values of $A_{i}$ for $i \in I^{*}$, we will assume that for each $i \in I$, the matrix $A_{i}$ has coefficients bounded below by some $\delta>0$. Note that, since $A_{i}$ is contractive, all of its coefficients are bounded above by 1 .

If $M_{1}, M_{2} \in \mathbb{R}^{2 \times 2}$ and $c \in \mathbb{R}$, by $M_{1}<M_{2}$ we mean that the inequality holds for each coefficient, and by $c<M_{1}$ we will mean that all coefficients of $M_{1}$ are strictly greater than $c$. In the same way we define $M_{1}>M_{2}$ and $c>M_{1}$. Note that if $0<M_{1}<M_{2}$, then $\alpha_{1}\left(M_{1}\right)<\alpha_{1}\left(M_{2}\right)$ by the Perron-Frobenius Theorem. Fix $0<\varepsilon<\delta$, and suppose that for each $i \in I$ there is a matrix $B_{i} \in \mathbb{R}^{2 \times 2}$ such that

$$
-\varepsilon<A_{i}-B_{i}<\varepsilon
$$


Let $\varepsilon_{1}=\varepsilon / \delta$, and note that

$$
\left(1-\varepsilon_{1}\right) A_{i}<B_{i}<\left(1+\varepsilon_{1}\right) A_{i} .
$$

Iterating this, we get that if $i \in I^{n}$, then

$$
\left(1-\varepsilon_{1}\right)^{n} A_{\mathrm{i}}<B_{\mathrm{i}}<\left(1+\varepsilon_{1}\right)^{n} A_{\mathrm{i}},
$$

and hence

$$
\left(1-\varepsilon_{1}\right)^{n} \alpha_{1}\left(A_{\dot{i}}\right)<\alpha_{1}\left(B_{\dot{i}}\right)<\left(1+\varepsilon_{1}\right)^{n} \alpha_{1}\left(A_{\dot{i}}\right) .
$$

A straightforward calculation shows that, for $i \in I$,

$$
\left|\operatorname{det}\left(A_{i}\right)\right|-8 \varepsilon<\left|\operatorname{det}\left(B_{i}\right)\right|<\left|\operatorname{det}\left(A_{i}\right)\right|+8 \varepsilon,
$$

whence, letting

$$
\varepsilon_{2}=\max _{i \in I} 8 \varepsilon\left|\operatorname{det}\left(A_{i}\right)\right|^{-1}
$$

we obtain

$$
\left(1-\varepsilon_{2}\right)\left|\operatorname{det}\left(A_{i}\right)\right|<\left|\operatorname{det}\left(B_{i}\right)\right|<\left(1+\varepsilon_{2}\right)\left|\operatorname{det}\left(A_{i}\right)\right| .
$$

Recall the definition of the pressure function given in (2.4). Let $P_{\mathcal{A}}$ and $P_{\mathcal{B}}$ denote the pressures corresponding to the matrices $\left\{A_{i}\right\}_{i \in I}$ and $\left\{B_{i}\right\}_{i \in I}$, respectively. Let $t$ be such that $P_{\mathcal{A}}(t)=0$, and let $s$ be such that $P_{\mathcal{B}}(s)=0$. Our goal is to show that $s \rightarrow t$ as $\varepsilon \downarrow 0$.

Let $D=\max _{i \in I}\left|\operatorname{det}\left(A_{i}\right)\right|$. Pick any $D^{\prime} \in(D, 1)$, and suppose $\varepsilon$ is so small that $D+8 \varepsilon<D^{\prime}$. If $s \geq 2$, then it is easy to see that the pressure is given by

$$
P_{\mathcal{B}}(s)=\log \left(\sum_{i \in I}\left|\operatorname{det}\left(B_{i}\right)\right|^{s / 2}\right) \leq \log \kappa-\frac{s}{2}\left|\log D^{\prime}\right| .
$$

Using this, we see that

$$
s \leq \max \left(2 \log \kappa /\left|\log D^{\prime}\right|, 2\right)=: T .
$$

Since, for $M \in \mathbb{R}^{2 \times 2}, \alpha_{2}(M)=|\operatorname{det}(M)| / \alpha_{1}(M)$, we obtain from (3.3) and the multiplicativity of the determinant that, for $i \in I^{n}$,

$$
\lambda_{1}^{n} \varphi^{s}\left(A_{\mathrm{i}}\right)<\varphi^{s}\left(B_{\mathrm{i}}\right)<\lambda_{2}^{n} \varphi^{s}\left(A_{\mathrm{i}}\right),
$$

where

$$
\begin{aligned}
& \lambda_{1}=\left(1-\varepsilon_{1}\right)\left(1+\varepsilon_{1}\right)^{-1}\left(1-\varepsilon_{2}\right)^{T / 2}, \\
& \lambda_{2}=\left(1+\varepsilon_{1}\right)\left(1-\varepsilon_{1}\right)^{-1}\left(1+\varepsilon_{2}\right)^{T / 2} .
\end{aligned}
$$

In order to see that (3.4) holds, it is convenient to consider the cases $0 \leq s<1$, $1 \leq s<2$, and $2 \leq s \leq T$ separately. From (3.4), we obtain

$$
P_{\mathcal{A}}(s)+\log \left(\lambda_{1}\right) \leq P_{\mathcal{B}}(s) \leq P_{\mathcal{A}}(s)+\log \left(\lambda_{2}\right),
$$

yielding

$$
P_{\mathcal{A}}(s) \in\left[-\log \left(\lambda_{2}\right),-\log \left(\lambda_{1}\right)\right] .
$$


Since $P_{\mathcal{A}}$ is a continuous, strictly decreasing function, so is its inverse $P_{\mathcal{A}}^{-1}$. But $\lambda_{1}, \lambda_{2} \rightarrow 1$ as $\varepsilon \rightarrow 0$, so the continuity of $P_{\mathcal{A}}^{-1}$ implies that $s \rightarrow t$ as $\varepsilon \downarrow 0$. This is exactly what we wanted to show.

\section{Proof of the main Result}

This section is dedicated to the proof of Theorem 3.3. We first collect several lemmas which will be used in the proof. These lemmas are geometric consequences of Definition 3.1. We remark that some of these lemmas are analogous to results in [10].

Lemma 4.1. Suppose that for each $i \in I$ there is a contractive invertible matrix $A_{i} \in \mathbb{R}^{2 \times 2}$ such that the conditions ( $(\mathrm{K} 1 \mathrm{a})$ and $(\overline{\mathrm{K} 1 \mathrm{~b}})$ are satisfied. Then $\left|A_{\mathrm{i}} x\right| \geq \cos (\beta) \alpha_{1}\left(A_{\mathrm{i}}\right)|x|$ for all $x \in X(\theta, \beta)$ and all $\mathrm{i} \in I^{*}$. Moreover, $\alpha_{1}\left(A_{\mathrm{ij}}\right) \geq$ $\cos ^{2}(\beta) \alpha_{1}\left(A_{\mathrm{i}}\right) \alpha_{1}\left(A_{\mathrm{j}}\right)$ whenever $\mathrm{i}, \mathrm{j} \in I^{*}$.

Proof. Let $\mathrm{i} \in I^{*}, x \in X(\theta, \beta)$, and write $x=x_{1} \theta_{1}\left(A_{\mathbf{i}}\right)+x_{2} \theta_{2}\left(A_{\mathbf{i}}\right)$. We may assume that $|x|=1$. Since $\theta_{1}\left(A_{\mathrm{i}}\right)$ is, by definition, the eigenvector of $A_{\mathrm{i}}^{*} A_{\mathrm{i}}$ corresponding to the largest eigenvalue, it follows from (K1a Perron-Frobenius Theorem that $\theta_{1}\left(A_{\mathrm{i}}\right) \in X(\theta, \beta)$ and $\left|x_{1}\right|=x \cdot \theta_{1}\left(A_{\mathrm{i}}\right) \geq \cos (\beta)$ (note that the Perron-Frobenius Theorem is usually stated for matrices preserving the positive cone, but it holds for any cone by a change of coordinates). Therefore

$$
\left|A_{\mathrm{i}} x\right|^{2}=\left|A_{\mathrm{i}}^{*} A_{\mathrm{i}} x \cdot x\right|=\alpha_{1}\left(A_{\mathrm{i}}\right)^{2} x_{1}^{2}+\alpha_{2}\left(A_{\mathrm{i}}\right)^{2} x_{2}^{2} \geq \alpha_{1}\left(A_{\mathrm{i}}\right)^{2} \cos ^{2}(\beta)
$$

giving the first claim.

The second claim follows immediately since

$$
\alpha_{1}\left(A_{\mathrm{ij}}\right) \geq\left|A_{\mathrm{i}} A_{\mathrm{j}} \theta\right| \geq \cos (\beta) \alpha_{1}\left(A_{\mathrm{i}}\right)\left|A_{\mathrm{j}} \theta\right| \geq \cos ^{2}(\beta) \alpha_{1}\left(A_{\mathrm{i}}\right) \alpha_{1}\left(A_{\mathrm{j}}\right)
$$

whenever $i, j \in I^{*}$.

Remark 4.2. Suppose that for each $i \in I$ there is a contractive invertible matrix $A_{i} \in \mathbb{R}^{2 \times 2}$ such that the conditions $(\overline{\mathrm{K} 1 \mathrm{a}})$ and $(\overline{\mathrm{K} 1 \mathrm{~b}})$ are satisfied. It follows immediately from Lemma 4.1 that there exists a constant $D \geq 1$ such that for every $t \geq 0$

$$
D^{-1} \varphi^{t}\left(A_{\mathrm{i}}\right) \varphi^{t}\left(A_{\mathrm{j}}\right) \leq \varphi^{t}\left(A_{\mathrm{ij}}\right)
$$

whenever $i, j \in I^{*}$. In fact, $D=\cos ^{-2}(\beta)$ works.

Lemma 4.3. Suppose that for each $i \in I$ there is a contractive invertible matrix $A_{i} \in \mathbb{R}^{2 \times 2}$ such that the conditions $(\underline{\mathrm{K} 1 \mathrm{a}})$ and $(\underline{\mathrm{K} 1 \mathrm{~b}})$ are satisfied. Then

(i) the angle between the vectors $A_{\mathrm{i}}\left(\theta_{1}\left(A_{\mathrm{i}}\right)\right)$ and $A_{\mathrm{i}} x$ is at most a constant times $\alpha_{2}\left(A_{\mathrm{i}}\right) / \alpha_{1}\left(A_{\mathrm{i}}\right)$ for every $\mathrm{i} \in I^{*}$ and $x \in X(\theta, \beta)$.

If in addition the condition ( $(\mathrm{K} 1 \mathrm{c})$ is satisfied, then

(ii) the angle between the vectors $A_{\mathrm{i}} x$ and $A_{\mathrm{j}} y$ is at least a constant times $\alpha_{2}\left(A_{\mathrm{i} \wedge \mathrm{j}}\right) / \alpha_{1}\left(A_{\mathrm{i} \wedge \mathrm{j}}\right)$ for every $\mathrm{i}, \mathrm{j} \in I^{*}$ and $x, y \in X(\theta, \beta)$. 
Proof. We first prove (i). Fix $i \in I^{*}$. Let $x \in S^{1} \cap X(\theta, \beta)$ and denote by $\gamma$ the (smaller) angle between $A_{\mathrm{i}} x$ and the major axis of the ellipse $A_{\mathrm{i}}(B(0,1))$, that is, the vector $A_{\mathrm{i}}\left(\theta_{1}\left(A_{\mathrm{i}}\right)\right)$. Since, by Lemma 4.1, we have $\left|A_{\mathrm{i}} x\right| \geq \cos (\beta) \alpha_{1}\left(A_{\mathrm{i}}\right)$, it follows that $|\sin (\gamma)| \leq \alpha_{2}\left(A_{\mathrm{i}}\right) /\left(\cos (\beta) \alpha_{1}\left(A_{\mathrm{i}}\right)\right)$. We conclude

$$
|\gamma| \leq \frac{\pi}{2}|\sin (\gamma)| \leq \frac{\pi}{2 \cos (\beta)} \alpha_{2}\left(A_{\mathrm{i}}\right) / \alpha_{1}\left(A_{\mathrm{i}}\right)
$$

Next we show (ii). Write $i=k i^{\prime}$ and $j=k j^{\prime}$, where $k=i \wedge j$, and notice that $i^{\prime}$ and $j^{\prime}$ start with different symbols. Therefore it follows from (K1) that there exists a constant $c>0$ (independent of $i$ and $j$ ) such that the angle between $A_{i^{\prime}} x$ and $A_{j^{\prime}} y$ is at least $c$ for any $x, y \in X(\theta, \beta)$. Hence it will be enough to prove the following claim: Given $c_{1}>0$ there is $c_{2}>0$ such that if $x, y \in S^{1} \cap X(\theta, \beta)$ and $|x-y| \geq c_{1}$, then the angle between $A_{\mathrm{k}} x$ and $A_{\mathrm{k}} y$ is at least $c_{2} \alpha_{2}\left(A_{\mathrm{k}}\right) / \alpha_{1}\left(A_{\mathrm{k}}\right)$ for all $\mathrm{k} \in I^{*}$.

To prove the claim consider the triangle with vertices $0, A_{\mathrm{k}} x, A_{\mathrm{k}} y$. Denote the angle at 0 by $\gamma$. By Lemma 4.1, the sides containing 0 have lengths between $\cos (\beta) \alpha_{1}\left(A_{\mathrm{k}}\right)$ and $\alpha_{1}\left(A_{\mathrm{k}}\right)$, while by the assumption, the length of the third side is at least $c_{1} \alpha_{2}\left(A_{\mathrm{k}}\right)$. We compute the area of the triangle in two ways. On the one hand, it is $\left|A_{\mathrm{k}} x\right|\left|A_{\mathrm{k}} y\right| \sin (\gamma) / 2 \leq \alpha_{1}\left(A_{\mathrm{k}}\right)^{2} \sin (\gamma) / 2$. Since one of the other two angles of the triangle must be at least $\pi / 6$ (otherwise $\gamma>2 \pi / 3$ and there is nothing to prove), the area of the triangle is also at least $\cos (\beta) c_{1} \alpha_{1}\left(A_{\mathrm{k}}\right) \alpha_{2}\left(A_{\mathrm{k}}\right) \sin (\pi / 6) / 2$. By comparing these two estimates, the claim follows. The proof is complete.

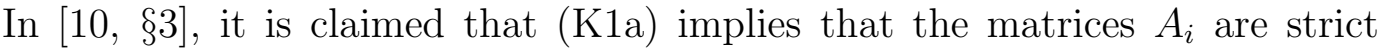
contractions acting on the space of lines through the origin with positive slope, where the metric is the smaller angle between them. This assertion is wrong, as the following example shows: let

$$
A=\left(\begin{array}{ll}
1 & \varepsilon \\
\varepsilon & \varepsilon
\end{array}\right) .
$$

Let $\ell$ be the line through the origin and $(\varepsilon, 1)$ and let $\ell^{\prime}$ be the line through the origin and $(2 \varepsilon, 1)$. Then a simple calculation shows that the angle between the lines $A \ell$ and $A \ell^{\prime}$ is of the order of $\varepsilon^{-1}$ times the angle between $\ell$ and $\ell^{\prime}$ as $\varepsilon \downarrow 0$. However, the next lemma, and in particular (4.1), shows that [10, Proposition $3.1]$ is still correct.

Lemma 4.4. Suppose that for each $i \in I$ there is a contractive invertible matrix $A_{i} \in \mathbb{R}^{2 \times 2}$ such that the condition $(\overline{\mathrm{K} 1 \mathrm{a}})$ is satisfied. Then there exist constants $C \geq 1$ and $0<\eta<1$ such that

$$
\alpha_{2}\left(A_{\mathrm{i}}\right) \leq C \eta^{|\mathrm{i}|} \alpha_{1}\left(A_{\mathrm{i}}\right)
$$

whenever $i \in I^{*}$. 
Proof. Let us first show that there exists $C_{0} \geq 1$ and $0<\eta<1$ such that

$$
A_{\mathrm{i}}(X(\theta, \beta)) \subset X\left(A_{\mathrm{i}} \theta /\left|A_{\mathrm{i}} \theta\right|, C_{0} \eta^{|\mathbf{i}|} \beta\right)
$$

whenever $i \in I^{*}$. Denote the space of all lines through the origin which are contained in $\overline{X(\theta, \beta)}$ by $\mathcal{P}(\theta, \beta)$. The smaller angle between any two lines $\ell_{1}, \ell_{2}$ will be denoted by $\Varangle\left(\ell_{1}, \ell_{2}\right)$. Since the maps $A_{i}$ are not necessarily contractions with respect to the metric $\Varangle$, we will make use of a different, but equivalent, metric. This metric is used in some proofs of the Perron-Frobenius Theorem, see for example [19, Lemma 3.4].

Let $\ell_{0}$ be a line through the origin which is not contained in $X(\theta, \beta)$, and such that $\Varangle\left(\ell_{0}, \ell\right)<\pi / 2$ for all $\ell \in \mathcal{P}(\theta, \beta)$. Define $d: \mathcal{P}(\theta, \beta)^{2} \rightarrow \mathbb{R}$ by setting

$$
d\left(\ell_{1}, \ell_{2}\right)=\left|\log \tan \left(\Varangle\left(\ell_{0}, \ell_{1}\right)\right)-\log \tan \left(\Varangle\left(\ell_{0}, \ell_{2}\right)\right)\right|
$$

as $\ell_{1}, \ell_{2} \in \mathcal{P}(\theta, \beta)$. It is easy to verify that $d$ is indeed a metric and, moreover, there is a constant $C_{0} \geq 1$ such that

$$
C_{0}^{-1 / 2} \Varangle\left(\ell_{1}, \ell_{2}\right) \leq d\left(\ell_{1}, \ell_{2}\right) \leq C_{0}^{1 / 2} \Varangle\left(\ell_{1}, \ell_{2}\right),
$$

for all $\ell_{1}, \ell_{2} \in \mathcal{P}(\theta, \beta)$. This is true since $\log \tan$ has a bounded derivative on a compact subset of $(0, \pi / 2)$. We claim that the maps $A_{i}$ acting on $\mathcal{P}(\theta, \beta)$ are uniformly contractive with respect to $d$. To prove this, we may fix $i \in I$ and assume that

$$
A_{i}=\left(\begin{array}{ll}
a & b \\
c & d
\end{array}\right)
$$

Moreover, after an appropriate rotation we can assume that $\ell_{0}$ is the $x$-axis, and all elements of $\mathcal{P}(\theta, \beta)$ have positive slope. Hence $a, b, c, d$ are nonzero and have the same sign. We will denote the slope of $\ell \in \mathcal{P}(\theta, \beta)$ by $s(\ell)$. After this normalization, we have

$$
d\left(A_{i} \ell_{1}, A_{i} \ell_{2}\right)=\left|\log \left(s\left(A_{i} \ell_{1}\right)\right)-\log \left(s\left(A_{i} \ell_{2}\right)\right)\right|,
$$

where

$$
s\left(A_{i} \ell\right)=\frac{c+d s(\ell)}{a+b s(\ell)}
$$

for any $\ell \in \mathcal{P}(\theta, \beta)$. In order to verify the claim, it suffices to show that the derivative of the function $g: \mathbb{R} \rightarrow \mathbb{R}, g(s)=\log \frac{c+d e^{s}}{a+b e^{s}}$, is strictly less than 1 in absolute value. It is straightforward to see that

$$
\left|g^{\prime}(s)\right|=\frac{|a d-b c| e^{s}}{\left(a+b e^{s}\right)\left(c+d e^{s}\right)}
$$

attains its maximum value at $s_{0}=\frac{1}{2} \log \frac{a c}{b d}$. Some elementary algebra shows that

$$
\left|g^{\prime}\left(s_{0}\right)\right|=\frac{|a d-b c|}{a d+b c+2 \sqrt{a b c d}}<1
$$

which is exactly what we wanted. 
Using the claim and (4.2), we see that there exists $0<\eta<1$ such that

$$
\Varangle\left(A_{\mathrm{i}} \ell_{1}, A_{\mathrm{i}} \ell_{2}\right)<C_{0} \eta^{|\mathrm{i}|} \Varangle\left(\ell_{1}, \ell_{2}\right),
$$

for any $i \in I^{*}$ and $\ell_{1}, \ell_{2} \in \mathcal{P}(\theta, \beta)$. Taking $\ell_{1}, \ell_{2}$ as the two lines which make up the boundary of $X(\theta, \beta)$, the assertion (4.1) follows.

To finally prove the lemma, notice that for each $i \in I^{*}$, we have

$$
\begin{aligned}
\mathcal{L}^{2}\left(A_{\mathrm{i}}(B(0,1) \cap X(\theta, \beta))\right) & =\mathcal{L}^{2}(B(0,1) \cap X(\theta, \beta)) \operatorname{det}\left(A_{\mathrm{i}}\right) \\
& =\beta \alpha_{1}\left(A_{\mathrm{i}}\right) \alpha_{2}\left(A_{\mathrm{i}}\right) .
\end{aligned}
$$

On the other hand, using (4.1), we have

$$
\begin{aligned}
\mathcal{L}^{2}\left(A_{\mathrm{i}}(B(0,1) \cap X(\theta, \beta))\right) & \leq \mathcal{L}^{2}\left(B\left(0, \alpha_{1}\left(A_{\mathrm{i}}\right)\right) \cap X\left(A_{\mathrm{i}} \theta /\left|A_{\mathrm{i}} \theta\right|, C_{0} \eta^{|\mathrm{i}|} \beta\right)\right) \\
& =C \eta^{|\mathbf{i}|} \beta \alpha_{1}\left(A_{\mathrm{i}}\right)^{2}
\end{aligned}
$$

for some constant $C \geq 1$. Comparing the two last displayed formulas yields the result.

Now we are ready to prove the main theorem.

Proof of Theorem 3.3. The upper bound $\overline{\operatorname{dim}}_{\mathrm{M}}(E) \leq t$ holds in general, for example, see [1] and [3]. Since (K2) implies $\operatorname{dim}_{\mathrm{H}}(E) \geq 1$, it is enough to prove that $\operatorname{dim}_{\mathrm{M}}(E) \geq t$. The continuity assertion will then follow from Lemma 3.4.

Recalling Remark 4.2, let $\mu, 1>\lambda_{1}(\mu) \geq \lambda_{2}(\mu)>0$, and $c, D \geq 1$ be as in Theorem 2.1. Fix $0<\varepsilon \leq \frac{1}{2} c^{-2} D^{-1} \underline{\alpha} \leq \frac{1}{2}$. Using Egorov's Theorem, we find an integer $n_{0}$ and a compact set $K \subset I^{\infty}$ so that $\mu\left(I^{\infty} \backslash K\right)<\varepsilon$ and

$$
\lambda_{k}(\mu)^{n(1+\varepsilon)} \leq \alpha_{k}\left(A_{\left.\mathrm{i}\right|_{n}}\right) \leq \lambda_{k}(\mu)^{n(1-\varepsilon)}
$$

whenever $i \in K, k \in\{1,2\}$, and $n \geq n_{0}$. Denoting

$$
\gamma_{k}=\frac{\log \lambda_{k}(\mu)}{\log \lambda_{1}(\mu) \lambda_{2}(\mu)^{t-1}}
$$

as $k \in\{1,2\}$, we notice that $\gamma_{1}+(t-1) \gamma_{2}=1$ and

$$
\varphi^{t}\left(A_{\left.\mathbf{i}\right|_{n}}\right)^{\gamma_{k}(1+\varepsilon) /(1-\varepsilon)} \leq \alpha_{k}\left(A_{\left.\mathbf{i}\right|_{n}}\right) \leq \varphi^{t}\left(A_{\left.\mathbf{i}\right|_{n}}\right)^{\gamma_{k}(1-\varepsilon) /(1+\varepsilon)}
$$

whenever $i \in K, k \in\{1,2\}$, and $n \geq n_{0}$. Since $\varepsilon$ can be arbitrarily small, (4.3) together with Lemma 4.4 imply that $\gamma_{1}<\gamma_{2}$.

For $r>0$ define

$$
Z(r)=\left\{\mathrm{i} \in I^{*}: \varphi^{t}\left(A_{\mathrm{i}}\right) \leq r<\varphi^{t}\left(A_{\mathbf{i}^{-}}\right)\right\},
$$


and notice that the set $Z(r)$ is incomparable for every $r>0$. Denote also $Z_{K}(r)=\{i \in Z(r):[i] \cap K \neq \emptyset\}$. Since

$$
\begin{aligned}
(c D)^{-1} \underline{\alpha} \sum_{i \in Z(r) \backslash Z_{K}(r)} r & \leq c^{-1} \sum_{i \in Z(r) \backslash Z_{K}(r)} \varphi^{t}\left(A_{\mathbf{i}}\right) \\
& \leq \sum_{i \in Z(r) \backslash Z_{K}(r)} \mu([i]) \leq \mu\left(I^{\infty} \backslash K\right)<\varepsilon,
\end{aligned}
$$

and, similarly,

$$
\sum_{i \in Z(r)} r \geq \sum_{i \in Z(r)} \varphi^{t}\left(A_{i}\right) \geq c^{-1}
$$

it follows that

$$
\# Z_{K}(r) \geq\left(c^{-1}-\varepsilon c D \underline{\alpha}^{-1}\right) r^{-1} \geq \frac{1}{2} c^{-1} r^{-1} .
$$

Hence, choosing $r>0$ small enough so that $|i| \geq n_{0}$ for every $i \in Z(r)$ and denoting $\xi=\min _{k \in\{1,2\}}\left(D^{-1} \underline{\alpha}\right)^{3 \gamma_{k}}$, it follows from (4.3) that

$$
\xi r^{\gamma_{k}(1+4 \varepsilon)} \leq \alpha_{k}\left(A_{\mathbf{i}}\right) \leq r^{\gamma_{k}(1-2 \varepsilon)}
$$

whenever $i \in Z_{K}(r)$ and $k \in\{1,2\}$.

Fix $i \in I^{*}$. Let $v_{\mathrm{i}}$ be the unit vector with direction equal to the major axis of the ellipse $A_{\mathbf{i}}(B(0,1))$. Explicitly, $v_{\mathbf{i}}=A_{\dot{i}}\left(\theta_{1}\left(A_{\dot{i}}\right)\right) / \alpha_{1}\left(A_{\dot{i}}\right)$. Since

$$
v_{\mathrm{i}} \cdot A_{\mathrm{i}} x=A_{\mathrm{i}}^{*} v_{\mathrm{i}} \cdot x=\alpha_{1}\left(A_{\mathrm{i}}\right) \theta_{1}\left(A_{\mathrm{i}}\right) \cdot x,
$$

for each $x \in E$, it follows from ( $(\overline{\mathrm{K} 2})$ that $\mathcal{L}^{1}\left(\left\{v_{\mathrm{i}} \cdot x: x \in E_{\mathrm{i}}\right\}\right) \geq \varrho \alpha_{1}\left(A_{\mathrm{i}}\right)$. Hence there exists a constant $T \geq 1$ so that for each $i \in I^{*}$ there is a rectangle $R_{\mathrm{i}}$ of size $\alpha_{1}\left(A_{\mathrm{i}}\right) \times \alpha_{2}\left(A_{\mathrm{i}}\right)$ with long side parallel to $A_{\mathrm{i}}\left(\theta_{1}\left(A_{\mathrm{i}}\right)\right)$ such that the $T \alpha_{2}\left(A_{\mathrm{i}}\right)$ neighborhood of $E_{\mathrm{i}}$ intersects $R_{\mathrm{i}}$ in a set of $\mathcal{L}^{2}$-measure at least $\varrho \alpha_{1}\left(A_{\mathrm{i}}\right) \alpha_{2}\left(A_{\mathrm{i}}\right)$.

Using Lemma 4.3)(ii) and (4.3), we get that there exists a constant $0<\omega^{\prime}<1$ such that if $i, j \in Z_{K}(r), i \neq j$, and $|i \wedge j| \geq n_{0}$, then the angle between the long sides of the rectangles $R_{\mathrm{i}}$ and $R_{\mathrm{j}}$, denoted by $\Varangle\left(R_{\mathrm{i}}, R_{\mathrm{j}}\right)$, is at least

$$
\omega^{\prime} \alpha_{2}\left(A_{\mathrm{i} \wedge \mathrm{j}}\right) / \alpha_{1}\left(A_{\mathrm{i} \wedge \mathrm{j}}\right) \geq \frac{\omega^{\prime} \varphi^{t}\left(A_{\mathrm{i} \wedge \mathrm{j}}\right)^{\gamma_{2}(1+\varepsilon) /(1-\varepsilon)}}{\varphi^{t}\left(A_{\mathrm{i} \wedge \mathrm{j}}\right)^{\gamma_{1}(1-\varepsilon) /(1+\varepsilon)}} \geq \omega^{\prime} r^{\gamma_{2}(1+4 \varepsilon)-\gamma_{1}(1-2 \varepsilon)} .
$$

If $|\mathrm{i} \wedge \mathrm{j}|<n_{0}$ then, using Lemma 4.3](ii) again,

$$
\Varangle\left(R_{\mathrm{i}}, R_{\mathrm{j}}\right) \geq \omega^{\prime} \alpha_{2}\left(A_{\mathrm{i} \wedge \mathrm{j}}\right) / \alpha_{1}\left(A_{\mathrm{i} \wedge \mathrm{j}}\right) \geq \omega^{\prime} \underline{\alpha}^{n_{0}} / \bar{\alpha}^{n_{0}} .
$$

Thus, in either case, if $i, j \in Z_{K}(r)$, $i \neq j$, then

$$
\Varangle\left(R_{\mathrm{i}}, R_{\mathrm{j}}\right) \geq \omega r^{\gamma_{2}(1+4 \varepsilon)-\gamma_{1}(1-2 \varepsilon)},
$$

where $\omega=\omega^{\prime} \underline{\alpha}^{n_{0}} / \bar{\alpha}^{n_{0}}<1$.

In order to apply Proposition [3.2, all the rectangles must have the same size. Let

$$
\alpha_{1}^{\prime}=r^{\gamma_{1}(1-2 \varepsilon)} \frac{r^{\gamma_{2}(1-2 \varepsilon)}}{\omega r^{\gamma_{2}(1+4 \varepsilon)}} \geq r^{\gamma_{1}(1-2 \varepsilon)}
$$


and let also $\alpha_{2}^{\prime}=r^{\gamma_{2}(1-2 \varepsilon)}$ (bear in mind that both $\alpha_{1}^{\prime}$ and $\alpha_{2}^{\prime}$ depend on $r$ ). It follows from (4.5) that each rectangle $R_{\mathrm{i}}$, with $\mathrm{i} \in Z_{K}(r)$, is contained in a rectangle $R_{\mathrm{i}}^{\prime}$ of size $\alpha_{1}^{\prime} \times \alpha_{2}^{\prime}$ with long side still parallel to $A_{\mathrm{i}}\left(\theta_{1}\left(A_{\mathrm{i}}\right)\right)$. Moreover, by (4.6), the angle between any two such rectangles is at least $\alpha_{2}^{\prime} / \alpha_{1}^{\prime}$.

Let $\delta=\operatorname{Tr}^{\gamma_{2}(1-2 \varepsilon)}$. We write $E(\delta)$ for the $\delta$-neighborhood of $E$. Using (4.5) once again, notice that, whenever $i \in Z_{K}(r), E(\delta)$ contains a $T \alpha_{2}\left(A_{\mathrm{i}}\right)$ neighborhood of $E_{\mathrm{i}} \subset E$. Hence $E(\delta)$ intersects each rectangle $R_{\mathrm{i}}$, and therefore also each rectangle $R_{\mathrm{i}}^{\prime}$, in a set of $\mathcal{L}^{2}$-measure at least

$$
\varrho \alpha_{1}\left(A_{\mathrm{i}}\right) \alpha_{2}\left(A_{\mathbf{i}}\right) \geq \varrho \xi r^{\gamma_{1}(1+4 \varepsilon)} \xi r^{\gamma_{2}(1+4 \varepsilon)}=\tau \alpha_{1}^{\prime} \alpha_{2}^{\prime},
$$

where

$$
\tau=\varrho \xi^{2} r^{\left(\gamma_{1}+\gamma_{2}\right)(1+4 \varepsilon)} \frac{\omega r^{\gamma_{2}(1+4 \varepsilon)}}{r^{\gamma_{1}(1-2 \varepsilon)} r^{\gamma_{2}(1-2 \varepsilon)} r^{\gamma_{2}(1-2 \varepsilon)}}=\varrho \xi^{2} \omega r^{6 \varepsilon\left(\gamma_{1}+2 \gamma_{2}\right)} .
$$

We can now apply Proposition 3.2 to the set $E(\delta)$ and the family $\left\{R_{\mathrm{i}}^{\prime}: \mathrm{i} \in\right.$ $\left.Z_{K}(r)\right\}$ to obtain, for every $r>0$ small enough, that

$$
\begin{aligned}
\mathcal{L}^{2}(E(\delta)) & \geq \frac{\# Z_{K}(r) \tau\left(\tau \alpha_{1}^{\prime} \alpha_{2}^{\prime}\right)}{2 \sqrt{2} \log \left(2 \pi \alpha_{1}^{\prime} / \alpha_{2}^{\prime}\right)} \\
& \geq \frac{\left(\frac{1}{2} c^{-1} r^{-1}\right)\left(\varrho \xi^{2} \omega r^{6 \varepsilon\left(\gamma_{1}+2 \gamma_{2}\right)}\right)\left(\varrho \xi^{2} r^{\left(\gamma_{1}+\gamma_{2}\right)(1+4 \varepsilon)}\right)}{2 \sqrt{2} \log \left(2 \pi \omega^{-1} r^{\gamma_{1}(1-2 \varepsilon)-\gamma_{2}(1+4 \varepsilon)}\right)} \\
& =\frac{(4 \sqrt{2} c)^{-1} \varrho^{2} \xi^{4} \omega r^{\gamma_{1}+\gamma_{2}-1+\varepsilon\left(10 \gamma_{1}+16 \gamma_{2}\right)}}{\log \left(2 \pi \omega^{-1} r^{\gamma_{1}-\gamma_{2}-\varepsilon\left(2 \gamma_{1}+4 \gamma_{2}\right)}\right)},
\end{aligned}
$$

where in the second displayed line we used (4.4). Recalling the definition of $\delta$, we estimate

$$
\begin{aligned}
& \underline{\operatorname{dim}}_{\mathrm{M}}(E)=\liminf _{\delta \downarrow 0}\left(2-\frac{\log \mathcal{L}^{2}(E(\delta))}{\log \delta}\right) \\
& \geq 2-\limsup _{r \downarrow 0}\left(\frac{\log \left((4 \sqrt{2} c)^{-1} \varrho^{2} \xi^{4} \omega r^{\gamma_{1}+\gamma_{2}-1+\varepsilon\left(10 \gamma_{1}+16 \gamma_{2}\right)}\right)}{\log \left(T r^{\gamma_{2}(1-2 \varepsilon)}\right)}\right.\left.-\frac{\log \log \left(2 \pi \omega^{-1} r^{\gamma_{1}-\gamma_{2}-\varepsilon\left(2 \gamma_{1}+4 \gamma_{2}\right)}\right)}{\log \left(T r^{\gamma_{2}(1-2 \varepsilon)}\right)}\right) \\
&=2-\frac{\gamma_{1}+\gamma_{2}-1+\varepsilon\left(10 \gamma_{1}+16 \gamma_{2}\right)}{\gamma_{2}(1-2 \varepsilon)},
\end{aligned}
$$

provided that $\gamma_{1}-\gamma_{2}-\varepsilon\left(2 \gamma_{1}+4 \gamma_{2}\right)<0$. By our earlier remark that $\gamma_{1}<\gamma_{2}$, this can be achieved by starting with a very small $\varepsilon>0$. Since $\gamma_{1}-1=(1-t) \gamma_{2}$, we conclude, by letting $\varepsilon \downarrow 0$, that

$$
\underline{\operatorname{dim}}_{\mathrm{M}}(E) \geq 2-\frac{\gamma_{1}+\gamma_{2}-1}{\gamma_{2}}=t
$$

as desired. 


\section{ON THE PROJECTION CONDITION}

Of all the conditions in the definition of a self-affine set of Kakeya type, the projection condition ( $\mathrm{K2}$ ) is the only one which cannot be checked directly. In this section we prove easily verifiable criteria which will be used to produce examples where ( $\underline{\mathrm{K} 2}$ ) holds.

We introduce some notation. Given a set $F \subset \mathbb{R}^{d}$ and $e \in \mathbb{R}^{d}$, we will denote

$$
F \cdot e=\{x \cdot e: x \in F\} .
$$

The convex hull of $F$ will be denoted by $\operatorname{conv}(F)$. Recall that a matrix $M \in \mathbb{R}^{\kappa \times \kappa}$ with nonnegative coefficients is irreducible if for all $1 \leq i, j \leq \kappa$ there is $n>0$ such that $M_{i j}^{n}>0$. Finally, the identity matrix on $\mathbb{R}^{2 \times 2}$ will be denoted by $\mathrm{Id}_{2}$.

We state two simple lemmas for later reference.

Lemma 5.1. If $\left\{\mathcal{I}_{\mathrm{i}}: \mathrm{i} \in I^{*}\right\}$ is a collection of closed intervals such that for any $i \in I^{*}$

$$
\bigcup_{i \in I} \mathcal{I}_{\mathrm{i} i}=\mathcal{I}_{\mathrm{i}}
$$

then

$$
\bigcap_{k=0}^{\infty} \bigcup_{i \in I^{k}} \mathcal{I}_{\mathbf{i}}=\mathcal{I}_{\varnothing}
$$

Proof. Immediate by induction.

Lemma 5.2. Suppose $\mathcal{I}_{1}, \ldots, \mathcal{I}_{\kappa}$ are closed intervals. If the adjacency matrix $M \in \mathbb{R}^{\kappa \times \kappa}$ defined as

$$
M_{i j}= \begin{cases}1, & \text { if } \mathcal{I}_{i} \cap \mathcal{I}_{j} \neq \emptyset \\ 0, & \text { otherwise, }\end{cases}
$$

is irreducible, then $\bigcup_{i=1}^{\kappa} \mathcal{I}_{i}$ is an interval.

Proof. Left to the reader.

The following proposition, which may be of independent interest, provides a simple criterion to guarantee that all the projections of a self-affine set are intervals. Even though our application will be in $\mathbb{R}^{2}$, we state the result for affine IFS's on $\mathbb{R}^{\kappa}$ since the proof is the same.

Proposition 5.3. Suppose that for each $i \in I$ there are a contractive invertible matrix $A_{i} \in \mathbb{R}^{\kappa \times \kappa}$ with $\left\|A_{i}\right\| \leq \bar{\alpha}<1$ and a translation vector $a_{i} \in \mathbb{R}^{\kappa}$. Assume the adjacency matrix $M \in \mathbb{R}^{\kappa \times \kappa}$ defined as

$$
M_{i j}= \begin{cases}1, & \text { if } \operatorname{conv}\left(E_{i}\right) \cap \operatorname{conv}\left(E_{j}\right) \neq \emptyset, \\ 0, & \text { otherwise, }\end{cases}
$$

is irreducible. Then $E \cdot e=\operatorname{conv}(E) \cdot e$ for all $e \in \mathbb{R}^{\kappa}$ and, in particular, $E \cdot e$ is an interval or a single point. 
Proof. We will repeatedly use the fact that the action of taking convex hulls commutes with affine maps. As a first instance of this, observe that for any $i \in I^{*}$,

$$
A_{\mathrm{i}}(\operatorname{conv}(E))+a_{\mathrm{i}}=\operatorname{conv}\left(E_{\mathrm{i}}\right),
$$

where

$$
a_{\mathrm{i}}=\sum_{n=1}^{|\mathrm{i}|} A_{\left.\mathrm{i}\right|_{n-1}} a_{i_{n}} .
$$

Let $D$ denote the Hausdorff distance. Notice that (5.1) implies

$$
D\left(\operatorname{conv}\left(E_{\mathrm{i}}\right), E_{\mathrm{i}}\right) \leq \alpha_{1}\left(A_{\mathrm{i}}\right) D(\operatorname{conv}(E), E) .
$$

Therefore

$$
\lim _{k \rightarrow \infty} D\left(\bigcup_{i \in I^{k}} \operatorname{conv}\left(E_{\mathrm{i}}\right), E\right)=0
$$

which in turn yields that

$$
E \cdot e=\bigcap_{k=1}^{\infty} \bigcup_{\mathrm{i} \in I^{k}} \operatorname{conv}\left(E_{\mathrm{i}}\right) \cdot e .
$$

Hence in order to prove the proposition it is enough to show that the family $\left\{\operatorname{conv}\left(E_{\mathrm{i}}\right) \cdot e: \mathrm{i} \in I^{*}\right\}$ verifies the hypothesis of Lemma 5.1 for all $e \in \mathbb{R}^{\kappa}$. We will do so by induction on $|\mathrm{i}|$. Denote $\mathcal{I}_{\mathrm{i}}=\operatorname{conv}\left(E_{\mathrm{i}}\right) \cdot e$ as $\mathrm{i} \in I^{*}$, and note that $\mathcal{I}_{i} \cap \mathcal{I}_{j} \neq \emptyset$ whenever $\operatorname{conv}\left(E_{i}\right) \cap \operatorname{conv}\left(E_{j}\right) \neq \emptyset$. Since the matrix $M$ was assumed to be irreducible, the hypothesis of Lemma 5.2 is met, whence $\mathcal{J}_{\varnothing}:=\bigcup_{i \in I} \mathcal{I}_{i}$ is an interval, and thus equal to its convex hull. On the other hand, since

$$
E \cdot e \subset \mathcal{J}_{\varnothing} \subset \operatorname{conv}(E) \cdot e=\operatorname{conv}(E \cdot e),
$$

we have $\operatorname{conv}\left(\mathcal{J}_{\varnothing}\right)=\operatorname{conv}(E) \cdot e$. Hence $\mathcal{J}_{\varnothing}=\operatorname{conv}(E) \cdot e$, and this settles the case $|\mathrm{i}|=0$. Now assume the case $|\mathrm{i}|=k$ has been proven, and let $\mathrm{i}$ be a symbol of length $k+1$. Write $\mathcal{J}_{\mathrm{i}}=\bigcup_{i \in I} \operatorname{conv}\left(E_{\mathrm{i} i}\right) \cdot e$ and $\mathrm{i}=j \mathrm{j}$, where $j \in I$ and $|j|=k$. Then

$$
\begin{aligned}
\mathcal{J}_{\mathrm{i}} & =\bigcup_{i \in I}\left(A_{j}\left(\operatorname{conv}\left(E_{\mathrm{j} i}\right)\right)+a_{j}\right) \cdot e=A_{j}\left(\bigcup_{i \in I} \operatorname{conv}\left(E_{\mathrm{j} i}\right)\right) \cdot e+a_{j} \cdot e \\
& =\left(\bigcup_{i \in I} \operatorname{conv}\left(E_{\mathrm{j} i}\right)\right) \cdot A_{j}^{*} e+a_{j} \cdot e .
\end{aligned}
$$

By the inductive hypothesis, this is an interval. On the other hand, $\mathcal{J}_{\text {i }}$ contains $E_{\mathrm{i}} \cdot e$ and is contained in $\operatorname{conv}\left(E_{\mathrm{i}}\right) \cdot e$, whence its convex hull must be $\operatorname{conv}\left(E_{\mathrm{i}}\right) \cdot e$. This shows that $\mathcal{J}_{i}=\mathcal{I}_{i}$, which is what we wanted to prove.

Proposition 5.3 is useful because one can check whether it holds by simply plotting the self-affine set $E$, say using a computer program. It also yields a very simple algebraic criterion which guarantees that all linear projections are 
stably intervals, as the next corollary shows. Given $x, y \in \mathbb{R}^{2}$, we will denote $[x, y]=\{\lambda x+(1-\lambda) y: 0 \leq \lambda \leq 1\}$ and $(x, y)=[x, y] \backslash\{x, y\}$. Furthermore, if $i \in I$ then with the notation $i^{\infty}$, we mean the symbol $(i, i, \ldots) \in I^{\infty}$.

Corollary 5.4. Suppose that for each $i \in I$ there are a contractive invertible matrix $A_{i} \in \mathbb{R}^{2 \times 2}$ with $\left\|A_{i}\right\| \leq \bar{\alpha}$ and a translation vector $a_{i} \in \mathbb{R}^{2}$. Denote by $E$ the invariant set of the affine IFS $\Phi=\left\{A_{i}+a_{i}\right\}_{i \in I}$ and let

$$
\begin{aligned}
& x_{i}=\pi\left(i 1^{\infty}\right)=a_{i}+\sum_{n=0}^{\infty} A_{i} A_{1}^{n} a_{1}, \\
& y_{i}=\pi\left(i \kappa^{\infty}\right)=a_{i}+\sum_{n=0}^{\infty} A_{i} A_{\kappa}^{n} a_{\kappa},
\end{aligned}
$$

as $i \in I$. If the adjacency matrix $M \in \mathbb{R}^{\kappa \times \kappa}$ defined as

$$
M_{i j}= \begin{cases}1, & \text { if }\left(x_{i}, y_{i}\right) \cap\left(x_{j}, y_{j}\right) \text { is a single point }, \\ 0, & \text { otherwise, }\end{cases}
$$

is irreducible, then for each affine IFS $\Phi^{\prime}$ sufficiently close to $\Phi$ there is a constant $\varrho>0$ such that $E^{\prime} \cdot e$ is an interval having length at least $\varrho$ for all $e \in \mathbb{R}^{2}$. Here $E^{\prime}$ is the invariant set of $\Phi^{\prime}$.

Proof. Denote by $M^{\prime}$ the adjacency matrix corresponding to the system $\Phi^{\prime}$. Since the property that $\left(x_{i}, y_{i}\right)$ intersects $\left(x_{j}, y_{j}\right)$ in a single point is stable, we see that $M_{i j}^{\prime} \geq M_{i j}$ if $\Phi^{\prime}$ is sufficiently close to $\Phi$. In particular, $M^{\prime}$ is irreducible whenever $M$ is. Thus it is enough to verify the result for the original system $\Phi$. It follows from the assumptions that $E$ is not contained in a line. Thus there exists $\varrho>0$ such that $\operatorname{conv}(E)$ contains a ball of radius $\varrho$. Since trivially $\left(x_{i}, y_{i}\right) \subset \operatorname{conv}\left(E_{i}\right)$, the proof is finished by Proposition 5.3 .

We next present a different, but also stable and easily checkable, condition that guarantees that the projection condition $(\underline{\mathrm{K} 2})$ is met. Let $\mathcal{Q}_{2}$ denote the family of all vectors $v \in \mathbb{R}^{2}$ with strictly positive coefficients and define a partial order $\prec$ on $\mathbb{R}^{2}$ by setting $x \prec y$ if and only if $y-x \in \mathcal{Q}_{2}$. With the notation $x \preceq y$ we mean that $x \prec y$ or $x=y$.

Lemma 5.5. Suppose that for each $i \in I$ there are a contractive invertible matrix $A_{i} \in \mathbb{R}^{2 \times 2}$ with $\left\|A_{i}\right\| \leq \bar{\alpha}$ and a translation vector $a_{i} \in \mathbb{R}^{2}$. If $A_{i}$ has strictly positive coefficients for all $i \in I$ and the points $x_{i}, y_{i}$ defined in (5.3) satisfy

$$
x_{i} \prec x_{i+1} \prec y_{i} \prec y_{i+1}
$$

whenever $i \in\{1, \ldots, \kappa-1\}$, then there is a constant $\varrho>0$ such that $E \cdot e$ contains an interval of length $\left(y_{\kappa}-x_{1}\right) \cdot e \geq \varrho$ for all $e \in \mathcal{Q}_{2}$.

Proof. The proof runs parallel to that of Proposition 5.3. Given $i \in I^{*}$, write

$$
\ell_{\mathrm{i}}=A_{\mathrm{i}}\left(\left[x_{1}, y_{\kappa}\right]\right)+a_{\mathrm{i}}
$$


where $a_{\mathrm{i}}$ is given by (5.2). We set $A_{\varnothing}=\mathrm{Id}_{2}$ and $a_{\varnothing}=(0,0)$. Observe that

$$
D\left(\ell_{\mathrm{i}}, E_{\mathrm{i}}\right) \rightarrow 0 \text { as }|\mathrm{i}| \rightarrow \infty,
$$

whence

$$
\lim _{k \rightarrow \infty} D\left(\bigcup_{\mathbf{i} \in I^{k}} \ell_{\mathbf{i}}, E\right)=0
$$

which in turn yields that

$$
E \cdot e \supset \bigcap_{k=1}^{\infty} \bigcup_{i \in I^{k}} \ell_{i} \cdot e .
$$

Thus we only need to prove that the family $\left\{\ell_{i} \cdot e: i \in I^{*}\right\}$ verifies the hypothesis of Lemma 5.1 for all $e \in \mathcal{Q}_{2}$. Denoting $\mathcal{I}_{\mathrm{i}}=\ell_{\mathrm{i}} \cdot e$ as $\mathrm{i} \in I^{*}$, we will prove by induction on $|\mathrm{i}|$ that

$$
\mathcal{I}_{\mathbf{i}}=\bigcup_{i \in I} \mathcal{I}_{\mathrm{i} i}=\left(A_{\mathrm{i}}\left(\left[x_{1}, y_{\kappa}\right]\right)+a_{\mathrm{i}}\right) \cdot e,
$$

for all $e \in \mathcal{Q}_{2}$. Consider the case $|\mathrm{i}|=0$ first. Note that, for $i \in I$,

$$
x_{i}=A_{i} x_{1}+a_{i}, \quad y_{i}=A_{i} y_{\kappa}+a_{i} .
$$

Hence $\ell_{i}=\left[x_{i}, y_{i}\right]$. From (5.4) we get that $x_{1} \preceq x_{i} \prec y_{i} \preceq y_{\kappa}$ for $i \in I$, whence

$$
\bigcup_{i \in I}\left[x_{i}, y_{i}\right] \cdot e \subset\left[x_{1}, y_{\kappa}\right] \cdot e .
$$

On the other hand, from (5.4) we see that $x_{i} \prec y_{i+1}$ and $x_{i+1} \prec y_{i}$. Since $x \cdot e<y \cdot e$ whenever $x \prec y$ and $e \in \mathcal{Q}_{2}$, we get

$$
\left[x_{i}, y_{i}\right] \cdot e \cap\left[x_{i+1}, y_{i+1}\right] \cdot e \neq \emptyset,
$$

whenever $i \in\{1, \ldots, \kappa-1\}$. From (5.6) and (5.7), and recalling that $\ell_{i}=\left[x_{i}, y_{i}\right]$, we get (5.5) in the case $|\mathrm{i}|=0$. The inductive step follows the same pattern as in Proposition 5.3. details are omitted.

\section{EXAMPLES AND REMARKS}

We are now ready to state easily checkable conditions which guarantee that an affine IFS is stably of Kakeya type. Explicit examples follow below. In the following theorem, we will use the convention that $[x, y]=[y, x]$ if $x>y$.

Theorem 6.1. Suppose that for each $i \in I$ there are a contractive invertible matrix $A_{i} \in \mathbb{R}^{2 \times 2}$ with $\left\|A_{i}\right\| \leq \bar{\alpha}$ and a translation vector $a_{i} \in \mathbb{R}^{2}$. Assume further that for each $i \in I$ there are real numbers $u_{i}, v_{i}, w_{i}, z_{i}>0$ such that

$$
A_{i}=\left(\begin{array}{cc}
u_{i} & v_{i} \\
w_{i} & z_{i}
\end{array}\right)
$$

and the following two conditions hold:

(X1) The intervals $\left[w_{i} / u_{i}, z_{i} / v_{i}\right]$ are pairwise disjoint for every $i \in I$. 
(X2) The affine IFS $\left\{A_{i}+a_{i}\right\}_{i \in J}$, where $J \subset I$ has cardinality at least 2 , verifies either the hypotheses of Corollary 5.4 or the hypotheses of Lemma 5.5.

Then the affine IFS $\Phi=\left\{A_{i}+a_{i}\right\}_{i \in I}$ is stably of Kakeya type. In particular, the Minkowski dimension of the invariant set is given by the zero of the pressure formula (2.4), and is continuous on a neighborhood of $\Phi$.

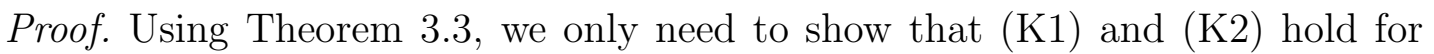
any small perturbation of $\Phi$. Since both (X1) and (X2) are stable properties, it is in fact enough to check that $\Phi$ is of Kakeya type.

Let $\theta=\frac{1}{\sqrt{2}}(1,1)$. Since the $A_{i}$ have strictly positive coefficients, both $A_{i}$ and $A_{i}^{*}$ map the cone $X(\theta, \pi / 2)$ into $X\left(\theta, \beta^{\prime}\right)$ for some $\beta^{\prime}<\pi / 2$. Hence there exists $\beta<\pi / 2$ such that both ( $\underline{\mathrm{K} 1 \mathrm{a}})$ and $(\underline{\mathrm{K} 1 \mathrm{~b}})$ hold.

Suppose that $(\underline{\mathrm{K} 1 \mathrm{C}})$ does not hold for $\Phi$. Then there is $s>0$ and $i, j \in I$ such that $i \neq j$ and

$$
(1, s)=A_{i}(x, y)=A_{j}\left(x^{\prime}, y^{\prime}\right),
$$

for some $x, y, x^{\prime}, y^{\prime}>0$. Some simple algebra shows that

$$
s=\frac{w_{i} x+z_{i} y}{u_{i} x+v_{i} y}=\frac{w_{j} x^{\prime}+z_{j} y^{\prime}}{u_{j} x^{\prime}+v_{j} y^{\prime}}
$$

whence $s \in\left[w_{i} / u_{i}, z_{i} / v_{i}\right] \cap\left[w_{j} / u_{j}, z_{j} / v_{j}\right]$, which contradicts (X1).

Let $F$ be the invariant set of $\Psi=\left\{A_{i}+a_{i}\right\}_{i \in J}$. It is clear that $F \subset E$. If $\Psi$ verifies the conditions of Corollary 5.4 , then $(\underline{\mathrm{K} 2})$ is immediately satisfied for $\Psi$ and hence also for $\Phi$. Likewise, if $\Psi$ satisfies the hypotheses of Lemma 5.5, then (K2) holds for $\Phi$. This is true since $\theta_{1}\left(A_{i}\right)$ has positive coordinates thanks to the Perron-Frobenius Theorem. The proof is complete.

We remark that finding an explicit neighborhood to which Theorem6.1 applies is an elementary, if tedious, exercise.

Example 6.2. We consider our first specific example. Let

$$
A_{1}(r, \varepsilon)=\left(\begin{array}{cc}
r & r+\varepsilon \\
\varepsilon & r
\end{array}\right), \quad A_{2}(r, \varepsilon)=\left(\begin{array}{cc}
r & \varepsilon \\
r+\varepsilon & r
\end{array}\right) .
$$

The affine IFS $\left\{A_{1}(r, 0)+a_{1}, A_{2}(r, 0)+a_{2}\right\}$ was studied in [2], where it is proven that the singularity dimension is 1 when $r=1 / 3$. This IFS does not verify (K1⿴囗十); however, $\left\{A_{1}(r, \varepsilon)+a_{1}, A_{1}(r, \varepsilon)+a_{2}\right\}$ does satisfy (X1), and hence (K1), for all small $\varepsilon>0$.

Figure1depicts the invariant set when $r=0.4, \varepsilon=0.1$ and the translations are $a_{1}=(-0.3,-0.3)$ and $a_{2}=-a_{1}$. For these values of the parameters the spectral radius of the matrices $A_{i}(r, \varepsilon)$ is approximately $0.624>1 / 2$; thus Falconer's Theorem does not apply. However, the conditions of Corollary 5.4 are clearly met (this can be verified algebraically without effort). Thus, by Theorem 6.1, this is stably a self-affine set of Kakeya-type. We remark that by picking appropriate 


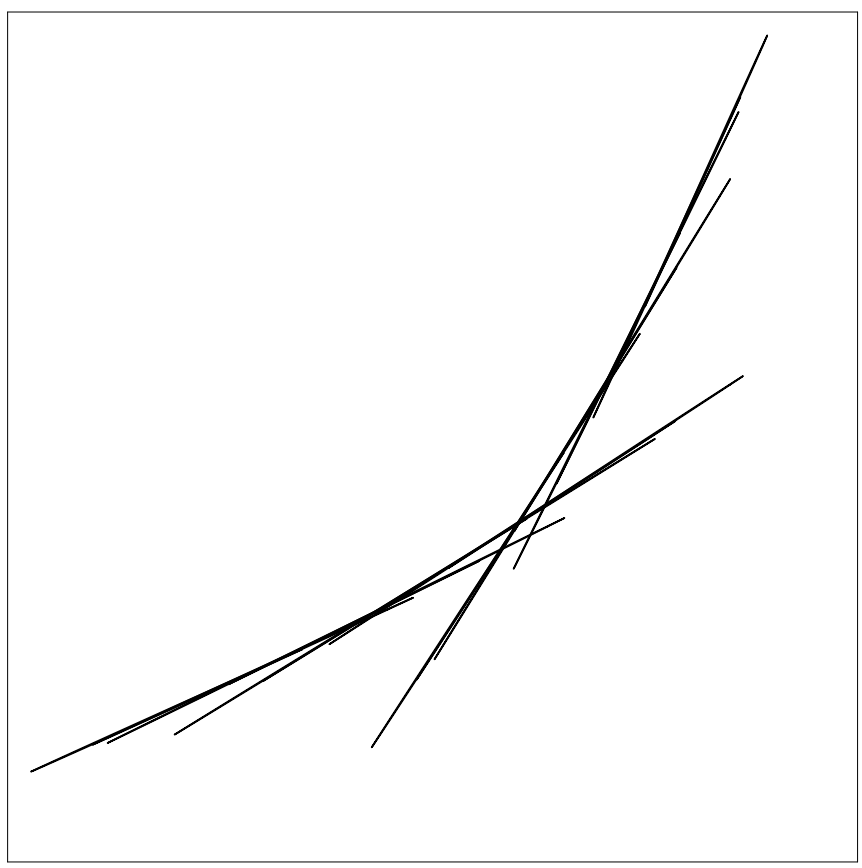

Figure 1: A self-affine set of Kakeya type.

values of $r$ and $\varepsilon$ one can obtain examples where the norms of the maps are arbitrarily close to 1 .

Notice that the invariant set resembles a union of approximately equally long segments pointing in different directions, underlining the Kakeya-type structure. Also observe that this particular example appears to be overlapping, although proving this rigorously looks very difficult.

Lemma 6.3. Suppose that for each $i \in\{1,2\}$ there is a contractive invertible matrix $A_{i} \in \mathbb{R}^{2 \times 2}$ with strictly positive coefficients and $\left\|A_{i}\right\| \leq \bar{\alpha}$, such that the condition (X1) is satisfied. Let

$$
B_{2}=\sum_{n=0}^{\infty} A_{2}^{n}=\left(I d_{2}-A_{2}\right)^{-1} .
$$

If both $A_{1} B_{2}-I d_{2}$ and $\left(I d_{2}-A_{1}\right) B_{2}$ have strictly positive coefficients, then for any vector $a_{2}$ with strictly positive coefficients, the affine IFS $\left\{A_{1}, A_{2}+a_{2}\right\}$ is stably of Kakeya type.

Proof. Notice that $B_{2}$ has strictly positive coefficients. The points defined in (5.3) are now $x_{1}=0, y_{1}=A_{1} B_{2} a_{2}, x_{2}=a_{2}$, and $y_{2}=B_{2} a_{2}$. Suppose $a_{2} \in \mathcal{Q}_{2}$. It is clear that $x_{1} \prec x_{2}$. Moreover, $x_{2} \prec y_{1}$ whenever $A_{1} B_{2}-\mathrm{Id}_{2}$ has strictly positive coefficients, and $y_{1} \prec y_{2}$ whenever $\left(\mathrm{Id}_{2}-A_{1}\right) B_{2}$ has strictly positive coefficients. 
Thus we have shown that the hypotheses of Lemma 5.5 hold, whence the lemma is immediate from Theorem 6.1.

Example 6.4. As a concrete example, let

$$
A_{1}=\left(\begin{array}{cc}
0.35 & 0.40 \\
0.30 & 0.35
\end{array}\right), \quad A_{2}=\left(\begin{array}{cc}
0.40 & 0.45 \\
0.45 & 0.50
\end{array}\right)
$$

A straightforward calculation shows that $A_{1} B_{2}-\mathrm{Id}_{2}$ and $\left(\operatorname{Id}_{2}-A_{1}\right) B_{2}$ have positive coefficients. Hence, by Lemma 6.3, the affine IFS $\left\{A_{1}, A_{2}+a_{2}\right\}$, as well as any small perturbation, is of Kakeya type for any $a_{2} \in \mathcal{Q}_{2}$. In particular, the Minkowski dimension of the invariant set of this IFS is constant for all $a_{2} \in \mathcal{Q}_{2}$.

Example 6.5. As a final example, we exhibit an affine IFS of Kakeya type with an arbitrary number of maps. Choose $\kappa \geq 3$ and let $A_{1}, A_{2}$ be as in Example 6.4. For $j \in\{3, \ldots, \kappa\}$, we define

$$
A_{j}=\left(\begin{array}{cc}
\frac{1}{2} & \frac{1}{2} \\
\frac{1}{3 j-1} & \frac{1}{3 j}
\end{array}\right) .
$$

Note that $\left\{A_{1}, \ldots, A_{\kappa}\right\}$ satisfies (X1). Thus Theorem 6.1, applied with $J=$ $\{1,2\}$, implies that for any $a_{2} \in \mathcal{Q}_{2}$ and any $a_{3}, \ldots, a_{\kappa} \in \mathbb{R}^{2}$, the affine IFS

$$
\left\{A_{1}, A_{2}+a_{2}, A_{3}+a_{3}, \ldots, A_{\kappa}+a_{\kappa}\right\}
$$

is stably of Kakeya type.

We finish the paper with some questions and remarks.

Remark 6.6. (1) Our techniques do not extend easily to higher dimensions. One source of technical difficulties is having to deal with more than two singular values, but the main obstruction is of course that the Kakeya conjecture is open for dimension $d \geq 3$, and no analogue of Proposition 3.2 is known. We remark, however, that Lemma 4.1 does hold, with the same proof, in higher dimensions, although one needs to replace the cone $X(\theta, \beta)$ by a cone which is, after a change of coordinates, $\mathcal{Q}_{d} \cup-\mathcal{Q}_{d}$. Here $\mathcal{Q}_{d}$ is the family of all vectors $v \in \mathbb{R}^{d}$ with strictly positive coefficients. Note that in $\mathbb{R}^{2}$ both classes of cones agree, but not in higher dimensions. This observation will be useful in the appendix.

(2) We do not know if our results hold for nonlinear perturbations of the affine IFS's we study. In studying nonlinear, nonconformal IFS's one usually needs to assume the so-called "1-bunching" condition, which guarantees that certain kind of bounded distortion holds, and therefore allows control of the shape of the cylinder sets; see for example [5]. For a linear map $A$, 1-bunching is equivalent to $\alpha_{2}(A)>\left(\alpha_{1}(A)\right)^{2}$. This is exactly Hypothesis 2 in [10] and, as remarked in \$3, it cannot hold in our setting. More specifically, 1-bunching appears to be necessary to extend Lemma 4.3 to nonlinear maps.

(3) Computing the singularity dimension of an arbitrary affine IFS is a very difficult problem. Recently Falconer and Miao [6] succeeded in finding a closed 
formula in the case all the matrices are upper triangular but, as they indicate, in general it is very hard to even obtain good numerical estimates. In our setting, one could use Lemma 4.1 to obtain rigorous upper and lower bounds, but the convergence is extremely slow.

(4) It would be of interest to find more general conditions for the validity

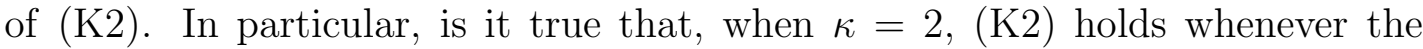
singularity dimension is strictly larger than 1 ?

(5) Falconer's Theorem shows that the equality of Hausdorff dimension and singular value dimension of a self-affine set is typical from the point of view of measure, at least when the norms of the linear maps do not exceed $\frac{1}{2}$, but does not say anything about the topological structure of the exceptional set. In every known counterexample, the linear parts of the affine maps commute; this is of course a nowhere dense condition. Our results provide some support to the conjecture that Minkowski dimension and singular value dimension agree for an open and dense family of affine IFS's.

Acknowledgement. PS wishes to thank Nuno Luzia and Boris Solomyak for helpful conversations and comments.

\section{Appendix A. Tractable SElF-AFFine Sets}

It was recently proved in 14 that the positivity of the Hausdorff measure is equivalent to a specific separation condition in a setting going beyond the conformal case. Working on $\mathbb{R}^{d}$, we define a nontrivial class of affine IFS's having this property. With the notation $\mathcal{H}^{t}$, we mean the $t$-dimensional Hausdorff measure, see [15, §4], and $\mathcal{Q}_{d}$ is the family of all vectors $v \in \mathbb{R}^{d}$ with strictly positive coefficients..

Definition A.1. If for each $i \in I$ there are a contractive invertible matrix $A_{i} \in \mathbb{R}^{d \times d}$ with $\left\|A_{i}\right\| \leq \bar{\alpha}<1$ and a translation vector $a_{i} \in \mathbb{R}^{d}$ then the collection of affine mappings $\left\{A_{i}+a_{i}: i \in I\right\}$ is called a tractable affine iterated function system and the invariant set $E \subset \mathbb{R}^{d}$ of this affine IFS a tractable selfaffine set provided that the condition (K1ia $)$ is satisfied for the cone $\mathcal{Q}_{d} \cup-\mathcal{Q}_{d}$ and the set $E$ is not contained in any hyperplane of $\mathbb{R}^{d}$.

We remark that we do not assume the separation condition ( $\mathrm{K1 \textrm {C }}$ ). To motivate the use of the cone $\mathcal{Q}_{d} \cup-\mathcal{Q}_{d}$, recall the explanation in Remark 6.6)(1). The condition on hyperplanes is simply a non-degeneracy assumption.

We shall show that on a tractable self-affine set the diameter of a cylinder is comparable to the corresponding largest singular value. For the proof, we need the following linear algebraic lemma.

Lemma A.2. Suppose there is a matrix $A \in \mathbb{R}^{d \times d}$ such that

$$
A\left(\overline{\mathcal{Q}_{d} \cup-\mathcal{Q}_{d}}\right) \subset \mathcal{Q}_{d} \cup-\mathcal{Q}_{d} .
$$


Then there exist a hyperplane $H$ such that for each $w \in \mathbb{R}^{d} \backslash H$ there is $n_{0} \in \mathbb{N}$ with $A^{n} w \in \mathcal{Q}_{d} \cup-\mathcal{Q}_{d}$ whenever $n \geq n_{0}$.

Proof. Let $\left\{\lambda_{1}, \ldots, \lambda_{d}\right\}$ be the spectrum of $A$, where $\left|\lambda_{1}\right| \geq \ldots \geq\left|\lambda_{n}\right|$. By the Perron-Frobenius Theorem, $\lambda_{1}$ is real and positive, and $\lambda_{1}>\left|\lambda_{2}\right|$. Moreover, if $v$ is the Perron eigenvector associated to $\lambda_{1}$ then $v \in \mathcal{Q}_{d} \cup-\mathcal{Q}_{d}$. Let $H$ be the hyperplane spanned by all the other eigenvectors of $A$ (this is a well-defined hyperplane since $\lambda_{1}$ is a simple, real eigenvalue). Note that $H$ is invariant under A.

Fix $w \in \mathbb{R}^{d} \backslash H$ and write $w=w_{1} v+h$, where $w_{1} \neq 0$ and $h \in H$. We have

$$
A^{n} w=w_{1} \lambda_{1}^{n} v+\left(\left.A\right|_{H}\right)^{n}(h)=\lambda_{1}^{n}\left(w_{1} v+\lambda_{1}^{-n}\left(\left.A\right|_{H}\right)^{n}(h)\right)
$$

for every $n \in \mathbb{N}$. Choose $\left|\lambda_{2}\right|<\mu<\lambda_{1}$ and $\delta$ small enough so that $B\left(w_{1} v, \delta\right) \subset$ $\mathcal{Q}_{d} \cup-\mathcal{Q}_{d}$. Since the spectral radius of $\left.A\right|_{H}$ is $\left|\lambda_{2}\right|$, we find $n_{0} \in \mathbb{N}$ such that $\left|\left(\left.A\right|_{H}\right)^{n}(h)\right|<\mu^{n}|h|$ and $\left(\mu / \lambda_{1}\right)^{n}|h|<\delta$ whenever $n \geq n_{0}$. Recalling (‥1), we conclude that $A^{n} w \in \mathcal{Q}_{d} \cup-\mathcal{Q}_{d}$ for $n \geq n_{0}$. The proof is finished.

Lemma A.3. Suppose the collection of affine mappings $\left\{A_{i}+a_{i}: i \in I\right\}$ is a tractable affine IFS. Then there exists a constant $C \geq 1$ such that

$$
C^{-1} \alpha_{1}\left(A_{\mathrm{i}}\right) \leq \operatorname{diam}\left(E_{\mathrm{i}}\right) \leq C \alpha_{1}\left(A_{\mathrm{i}}\right)
$$

whenever $i \in I^{*}$.

Proof. The diameter of $E_{\mathrm{i}}$ is at most a constant times $\alpha_{1}\left(A_{\mathrm{i}}\right)$ in general, so we only need to prove the other direction. Fix $i \in I$ and let $H$ be the hyperplane given by Lemma A.2 applied to the matrix $A_{i}$. By the tractability, the self-affine set $E$ is not contained in any translate of $H$. Therefore, the arithmetic difference $E-E$ is not contained in $H$ and we can find two different points $x, y \in E$ such that $y-x \notin H$. Applying Lemma $\underline{\text { A.2. }}$, we find $n$ such that $y^{\prime}-x^{\prime} \in X(\theta, \beta)$, where

$$
y^{\prime}=\left(A_{i}+a_{i}\right)^{n} y \in E, \quad x^{\prime}=\left(A_{i}+a_{i}\right)^{n} x \in E .
$$

By Remark 6.6(1) and Lemma 4.1, there exists a constant $\delta>0$ such that

$$
\operatorname{diam}\left(E_{\mathrm{i}}\right) \geq\left|A_{\mathrm{i}} y^{\prime}-A_{\mathrm{i}} x^{\prime}\right| \geq \delta\left|y^{\prime}-x^{\prime}\right| \alpha_{1}\left(A_{\mathrm{i}}\right) .
$$

The proof is complete.

We introduce in the following definition a natural separation condition to be used on tractable self-affine sets. Given a tractable affine IFS, define for $r>0$

$$
\left.Z(r)=\left\{\mathrm{i} \in I^{*}: \operatorname{diam}\left(E_{\mathrm{i}}\right) \leq r<\operatorname{diam}\left(E_{\mathrm{i}^{-}}\right)\right)\right\}
$$

and if in addition $x \in E$, set

$$
Z(x, r)=\left\{\mathrm{i} \in Z(r): E_{\mathrm{i}} \cap B(x, r) \neq \emptyset\right\} .
$$


Definition A.4. We say that a tractable self-affine set $E$ satisfies a ball condition if there exists a constant $0<\delta<1$ such that for each $x \in E$ there is $r_{0}>0$ such that for every $0<r<r_{0}$ there exists a set $\left\{x_{\mathrm{i}} \in \operatorname{conv}\left(E_{\mathrm{i}}\right): \mathrm{i} \in Z(x, r)\right\}$ such that the collection $\left\{B\left(x_{i}, \delta r\right): i \in Z(x, r)\right\}$ is disjoint. If $r_{0}>0$ above can be chosen to be infinity for every $x \in E$ then the tractable self-affine set $E$ is said to satisfy a uniform ball condition. Here with the notation $\operatorname{conv}(A)$, we mean the convex hull of a given set $A$.

Now we are ready to prove our result concerning tractable self-affine sets.

Theorem A.5. Suppose $E$ is a tractable self-affine set and $P(t)=0$ for some $0<t \leq 1$. Then $E$ satisfies the (uniform) ball condition if and only if $\mathcal{H}^{t}(E)>0$.

Proof. Notice first that if $0<t \leq 1$ then by Lemma A.3, the topological pressure defined in (2.4) is the same as the topological pressure defined in [14, (3.1)].

Observe that $0<\operatorname{diam}\left(E_{\mathrm{i}}\right) \leq \bar{\alpha}^{|\mathrm{i}|} \operatorname{diam}(E)$ for each $\mathrm{i} \in I^{*}$. According to Remark 6.6) (1) and Lemma 4.1, there is a constant $\delta>0$ for which $\alpha_{1}\left(A_{\mathrm{ij}}\right) \geq$ $\delta \alpha_{1}\left(A_{\mathbf{i}}\right) \alpha_{1}\left(A_{\mathrm{j}}\right)$ whenever $\mathrm{i}, \mathrm{j} \in I^{*}$. Hence, using Lemma A.3 again, we find a constant $D \geq 1$ such that

$$
D^{-1} \leq \frac{\operatorname{diam}\left(E_{\mathrm{ij}}\right)}{\operatorname{diam}\left(E_{\mathrm{i}}\right) \operatorname{diam}\left(E_{\mathrm{j}}\right)} \leq D
$$

for every $\mathrm{i}, \mathrm{j} \in I^{*}$. Since $E_{\mathrm{i} i} \subset E_{\mathrm{i}}$ as $\mathrm{i} \in I^{*}$ and $i \in I$, we have shown that the collection of compact sets $\left\{E_{\mathrm{i}}: \mathrm{i} \in I^{*}\right\}$ satisfies the assumptions (M1)-(M3) introduced in [14, $\S 3]$.

Using Lemma A.3 once again, we see that there exists a constant $C \geq 1$ such that $\left|A_{\mathrm{i}} x-A_{\mathrm{i}} y\right| \leq C \operatorname{diam}\left(E_{\mathrm{i}}\right)|x-y|$ for every $x, y \in E$ and for each $\mathrm{i} \in I^{*}$. Therefore, by [14, Lemma 5.1] and [14, Corollary 3.10], the proof is finished.

\section{REFERENCES}

[1] A. Douady and J. Oesterlé. Dimension de Hausdorff des attracteurs. C. R. Acad. Sci. Paris Sér. A-B, 290(24):1135-1138, 1980.

[2] G. A. Edgar. Fractal dimension of self-affine sets: some examples. Rend. Circ. Mat. Palermo (2) Suppl., 28:341-358, 1988.

[3] K. J. Falconer. The Hausdorff dimension of self-affine fractals. Math. Proc. Cambridge Philos. Soc., 103(2):339-350, 1988.

[4] K. J. Falconer. The dimension of self-affine fractals II. Math. Proc. Cambridge Philos. Soc., 111(1):169-179, 1992.

[5] K. J. Falconer. Bounded distortion and dimension for nonconformal repellers. Math. Proc. Cambridge Philos. Soc., 115(2):315-334, 1994.

[6] K. J. Falconer and J. Miao. Dimensions of self-affine fractals and multifractals generated by upper-triangular matrices. 2007. preprint http://www-maths.mcs.st-andrews.ac.uk/ kenneth/FalMiao.pdf.

[7] K. J. Falconer and J. Miao. Exceptional sets for self-affine fractals. 2007. preprint http://www-maths.mcs.st-andrews.ac.uk/ kenneth/except10.pdf. 
[8] D.-J. Feng and Y. Wang. A class of self-affine sets and self-affine measures. J. Fourier Anal. Appl., 11(1):107-124, 2005.

[9] H. Hu. Box dimensions and topological pressure for some expanding maps. Comm. Math. Phys., 191(2):397-407, 1998.

[10] I. Hueter and S. P. Lalley. Falconer's formula for the Hausdorff dimension of a self-affine set in $\mathbb{R}^{2}$. Ergodic Theory Dynam. Systems, 15(1):77-97, 1995.

[11] J. E. Hutchinson. Fractals and self-similarity. Indiana Univ. Math. J., 30(5):713-747, 1981.

[12] T. Jordan, M. Pollicott, and K. Simon. Hausdorff dimension for randomly perturbed selfaffine attractors. Comm. Math. Phys., 270(2):519-544, 2007.

[13] A. Käenmäki. On natural invariant measures on generalised iterated function systems. Ann. Acad. Sci. Fenn. Math., 29(2):419-458, 2004.

[14] A. Käenmäki and M. Vilppolainen. Separation conditions on controlled Moran constructions. 2006. preprint http://www.math.jyu.fi/ ${ }^{\sim}$ antakae/publications/index.php.

[15] P. Mattila. Geometry of Sets and Measures in Euclidean Spaces: Fractals and Rectifiability. Cambridge University Press, Cambridge, 1995.

[16] R. D. Mauldin and M. Urbański. Dimensions and measures in infinite iterated function systems. Proc. London Math. Soc., 73(3):105-154, 1996.

[17] C. McMullen. The Hausdorff dimension of general Sierpiński carpets. Nagoya Math. J., 96:1-9, 1984.

[18] P. A. P. Moran. Additive functions of intervals and Hausdorff measure. Proc. Cambridge Philos. Soc., 42:15-23, 1946.

[19] M. Pollicott and M. Yuri. Dynamical systems and ergodic theory, volume 40 of London Mathematical Society Student Texts. Cambridge University Press, Cambridge, 1998.

[20] P. Shmerkin. Overlapping self-affine sets. Indiana Univ. Math. J., 55(4):1291-1331, 2006.

[21] P. Shmerkin and B. Solomyak. Zeros of $\{-1,0,1\}$ power series and connectedness loci for self-affine sets. Experiment. Math., 15(4):499-511, 2006.

[22] B. Solomyak. Measure and dimension for some fractal families. Math. Proc. Cambridge Philos. Soc., 124(3):531-546, 1998.

[23] J. M. Steele. Kingman's subadditive ergodic theorem. Ann. Inst. H. Poincaré Probab. Statist., 25(1):93-98, 1989.

[24] R. Temam. Infinite-dimensional dynamical systems in mechanics and physics. SpringerVerlag, New York, 1988.

[25] T. Wolff. Recent work connected with the Kakeya problem. Prospects in mathematics (Princeton, NJ, 1996), pages 129-162, 1999.

Department of Mathematics and Statistics, P.O. Box 35 (MaD), FI-40014 UniVERSITY OF JYVÄSKYLÄ, FiNLAND

E-mail address: antakae@maths.jyu.fi

E-mail address: shmerkin@maths.jyu.fi 